International Journal of Pure and Applied Mathematics

Volume 111 No. 3 2016, 429-453

ISSN: 1311-8080 (printed version); ISSN: 1314-3395 (on-line version)

url: http://www.ijpam.eu

doi: 10.12732 /ijpam.v111i3.8

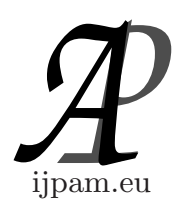

\title{
A SYSTEM OF DIFFERENCE EQUATIONS OF 8-TH ORDER FOR SOME MEJER'S FUNTIONS
}

\author{
L.A. Gutnik \\ Higher School of Economics \\ National Research University \\ Moscow, 10100, RUSSIAN FEDERATION
}

\begin{abstract}
In [4] we've considered a difference system connected with some Mejer's funtions, which satisfy to a differential equation of fourth order. Arithmetical applications $f$ these considerations can be found in [5], [6]. Here we analogously deduce a difference system for some Mejer's functions, which satisfy to a differential equation of eighth order.
\end{abstract}

In memory of Professor A.A. Buchstab.

AMS Subject Classification: 39AXX, 11B37, 33CXX

Key Words: difference system, Mejer's functions

\section{1. ?? Introduction. Formulation of Theorem ??.1}

We fix $\alpha \in \mathbb{N}_{0}$. As usually, $m_{\alpha}:=\Gamma(m+\alpha) / \Gamma(m)$ denotes Pochhammer's symbol. Let $|z|>1,-3 \pi / 2<\arg (z) \leq \pi / 2, \log (z)=\ln (|z|)+i \arg (z)$,

$$
\begin{gathered}
l=0,1,2, \nu \in \mathbb{N}_{0}, g_{l, 1}(s)=g_{l, 1}(s, \alpha, z, \nu)= \\
\left(-(-1)^{l} z\right)^{s} \Gamma(-s)(\Gamma(1+s))^{-3-2 l}(\Gamma(1+\nu+s))^{2+2 l}(\Gamma(1+\nu+\alpha-s))^{2+2 l},
\end{gathered}
$$

Received: $\quad$ October 8, 2016

Revised: $\quad$ November 12, 2016

Published: December 17, 2016 (c) 2016 Academic Publications, Ltd.

url: www.acadpubl.eu 


$$
\begin{aligned}
& f_{\alpha, l, 1}^{\vee}(z, \nu)=f_{\alpha, l, 1}(z, \nu)=-(-1)^{\nu l} \times \text { Mejer's function }
\end{aligned}
$$

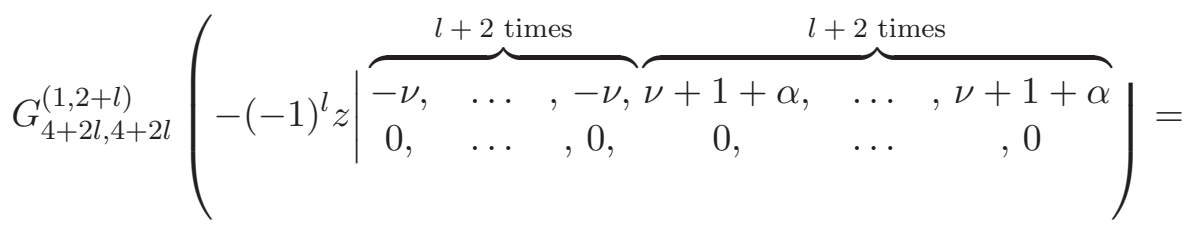

$$
\begin{aligned}
& -(-1)^{l \nu} \int_{L_{1}} \frac{g_{l, 1}(s)}{2 i \pi} d s, \text { where the curve } L_{1} \text { passes from }+\infty \text { to }+\infty
\end{aligned}
$$

in the negative direction such that the set $\mathbb{N}_{0}$ lies to the right from it, but the set $(-\infty,-1) \cap \mathbb{Z}$ lies to the left from it. All unremovable singular points of $g_{l, 1}(s)$, encircled by the curve $L_{1}$, are the points $s=0, \ldots, \nu+2 \alpha$, each of them is a pole of the first order, and $\operatorname{Res}\left(g_{l, 1}(s) ; k\right)=\lim _{s \rightarrow k}\left((s+k) g_{l, 1}(s)\right)$, where $l=0,1,2$ and $k=0, \ldots, \nu$. Let $s=k+u$,

$$
\begin{gathered}
H_{l, 1}(u, k, \nu)=g_{l, 1}(s)(k+u)=\left(-(-1)^{l} z\right)^{k+u} \Gamma(-k-u)(\Gamma(1+k+u))^{-3-2 l} \times \\
\left(\frac{\Gamma(1+\nu+k+u)}{\Gamma(1+\nu+\alpha-k-u)}\right)^{2+2 l}=\left(\prod_{\kappa=1}^{k}(-k+\kappa-u)^{-1}\right)\left(-(-1)^{l} z\right)^{k+u} \times \\
\Gamma(1-u)(\Gamma(1+k+u))^{-3-2 l}((\Gamma(1+\nu+k+u)) / \Gamma(1+\nu+\alpha-k-u))^{2+l},
\end{gathered}
$$

where $l=0,1,2$ and $k \in[0,+\infty) \cap \mathbb{Z}$. Hence,

$$
\begin{gathered}
\left((\nu+1)_{\alpha}\right)^{2+l} \operatorname{Res}\left(g_{l, 1}(s) ; k\right)=((\nu+\alpha) ! / \nu !)^{2+l} \lim _{u \rightarrow 0}\left(u g_{4+2 l, 4+2 l}^{(1,2+l)}(k+u)\right)= \\
-(-1)^{l k}\left(\frac{(\nu+\alpha) !(\nu+k) !}{\nu !(k !)^{2}(\nu+\alpha-k) !}\right)^{2+l}=-(-1)^{l k} z^{k}\left(\left(\begin{array}{c}
\nu+\alpha \\
k
\end{array}\right)\left(\begin{array}{c}
\nu+k \\
k
\end{array}\right)\right)^{2+2 l},
\end{gathered}
$$

where $l=0,1,2$ and $k=0, \ldots, \nu$. Consequently,

$$
\begin{gathered}
f_{\alpha, l, 1}^{* \vee}(z, \nu):=f_{\alpha, l, 1}^{*}(z, \nu):=((\nu+\alpha) ! / \nu !)^{2+l} f_{\alpha, l, 1}(z, \nu)= \\
\left.\left((\nu+1)_{\alpha}\right)\right)^{2+l} f_{\alpha, l, 1}(z, \nu)=\sum_{k=0}^{\nu+\alpha}(-1)^{(\nu+k) l}(z)^{k}\left(\begin{array}{c}
\nu+\alpha \\
k
\end{array}\right)^{2+l}\left(\begin{array}{c}
\nu+k \\
\nu
\end{array}\right)^{2+l}
\end{gathered}
$$

where $l=0,1,2, \nu \in \mathbb{N}_{0}$. Let $\kappa \in[3,6] \cap \mathbb{Z}, k \in\{2,3\}$ for $l=0, k \in 2,3,5$ for $l=1, k \in\{2,3,5,7\}$ for $l=2, g_{l, \kappa}^{\sim}=g_{l, \kappa}^{\sim}(s)=g_{l, \kappa}^{\sim}(s)(s, \alpha, z, \nu)=$

$$
\left((-1)^{\kappa} z\right)^{s}(\Gamma(-s))^{\kappa+l}(\Gamma(1+s))^{-l-4+\kappa}(\Gamma(1+\nu+s) / \Gamma(1+\nu+\alpha,-s))^{2+l} .
$$




$$
\begin{aligned}
& f_{\alpha, l, \max (2,2 \kappa-5)}^{\vee}(z, \nu)=(-1)^{l \nu+\kappa} \times \text { Mejer's function }
\end{aligned}
$$

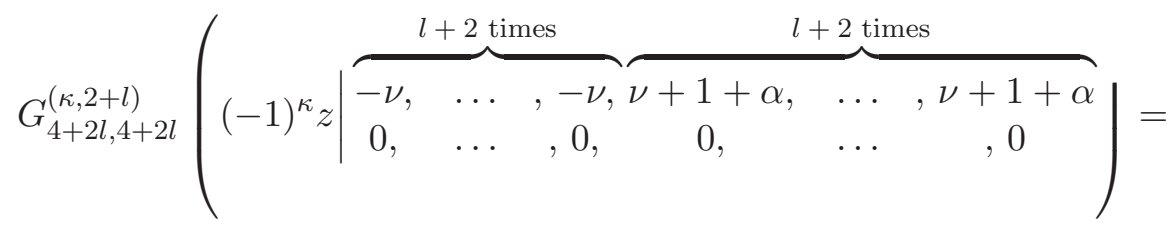

$$
\begin{aligned}
& (-1)^{l \nu+\kappa} \int_{L_{2}} \frac{g_{l, \kappa(s)}^{\sim}}{2 i \pi} d s, \text { where } \kappa \in[3,4+l] \cap \mathbb{Z}, \text { and the curve } L_{2} \text { passes }
\end{aligned}
$$

from $-\infty$ to $-\infty$ in the positive direction such that $\mathbb{Z} \cap(-\infty,-1]$ lies to the left from it, but $\mathbb{N}_{0}$ lies to the right from it. We put $f_{\alpha, l, k}(z, \nu):=f_{\alpha, l, k}^{\vee}(z, \nu)$ for $k \in\{2,3\}$. All unremovable singular points of $g_{l, \kappa}^{\sim}(s)$, encircled by $L_{2}$, compose the set $\left\{-1-\nu-k: k \in \mathbb{N}_{0}\right\}$, and we put $s=-\nu-1-k+u$. Then

$$
\begin{gathered}
g_{l, \kappa}^{\sim}(-\nu-1-k+u)=\left((-1)^{\kappa} z\right)^{-\nu-1-k+u} \times \\
(\Gamma(\nu+1+k-u))^{\kappa+l}(\Gamma(-\nu-k+u))^{-l-4+\kappa}(\Gamma(-k+u) / \Gamma(2+2 \nu+\alpha+k-u))^{2+l}= \\
\left((-1)^{\kappa} z\right)^{-\nu-1-k+u}(-1)^{(\nu+k)(l+\kappa)+k l}(\pi / \sin (u \pi))^{\kappa-2} \times \\
\left((\Gamma(\nu+1+k-u))^{2} /(\Gamma(1+k-u) \Gamma(2+2 \nu+\alpha+k-u))\right)^{2+l} .
\end{gathered}
$$

Let

$$
\begin{gathered}
H_{l}^{*}(\alpha, u, k, \nu)=\left(\frac{(\Gamma(\nu+1+k-u))^{2}}{\Gamma(1+k-u) \Gamma(2+2 \nu+\alpha+k-u)}\right)^{2+l}, \\
H_{l}^{* *}(z, \kappa, \alpha, u, k, \nu)=\left((-1)^{\kappa} z\right)^{-\nu-1-k+u}(-1)^{(\nu+k)(l+\kappa)+k l} H_{l}^{*}(\alpha, u, k, \nu), \\
R(\alpha, t, \nu)=\left(\prod_{j=1}^{\nu}(t-j)\right)^{/} \prod_{j=0 *}^{\nu+\alpha}(t+j), R(t, \nu)=R(1, t, \nu) .
\end{gathered}
$$

where $\nu \in \mathbb{N}_{0}$. Clearly,

$$
\begin{gathered}
H_{l}^{*}(\alpha, u, k, \nu)=R(\alpha, \nu+1+k-u, \nu)^{2+l} \\
\frac{\partial}{\partial u} H_{l}^{*}(\alpha, u, k, \nu)=-\frac{\partial}{\partial t}\left(R^{2+l}\right)(\alpha, \nu+1+k-u, \nu),
\end{gathered}
$$

where $\nu \in \mathbb{N}_{0}$. In view of (4) - (8),

$$
\tilde{g_{l, \kappa}}(-\nu-1-k+u)=\left(\frac{\pi}{\sin (u \pi)}\right)^{\kappa-2} H_{l}^{* *}(z, \kappa, \alpha, u, k, \nu) .
$$


In view of $(10),(6)$ the function $g_{l, \kappa}^{\sim}(s)$ has at he point $-\nu-1-k$ the pole of the order $\kappa-2$. Therefore, in view of (10), (6),

$$
\begin{gathered}
\operatorname{Res}\left(\tilde{g}_{l, \kappa}^{\sim}(s) ;-1-\nu-k\right)=1 /(\kappa-3) ! \times \\
\lim _{u \rightarrow 0}\left(\left(\frac{\partial}{\partial u}\right)^{\kappa-3}\left(u^{\kappa-2} g_{4+2 l, 4+2 l}^{(\kappa+l, 2+l)}(-1-\nu-k+u)\right)\right)=\frac{1}{(\kappa-3) !} \times \\
\lim _{u \rightarrow 0}\left(\left(\frac{\partial}{\partial u}\right)^{\kappa-3}\left(\frac{u \pi}{\sin (u \pi)}\right)^{\kappa-2} H_{l}^{* *}(z, \kappa, \alpha, u, k, \nu)\right) .
\end{gathered}
$$

In view of $(9),(6)$,

$$
\begin{gathered}
\lim _{u \rightarrow 0}\left(\left(\frac{\partial}{\partial u}\right)^{\eta} H_{l}^{* *}(z, \kappa, \alpha, u, k, \nu)\right)=z^{-1-\nu-k} \times \\
(-1)^{l \nu+\kappa} \sum_{\sigma=0}^{\eta}\left(\begin{array}{l}
\eta \\
\sigma
\end{array}\right)\left(\log \left((-1)^{\kappa} z\right)\right)^{\eta-\sigma}\left(\left(\frac{\partial}{\partial u}\right)^{\sigma} H_{l}^{*}\right)(\alpha, 0, k, \nu)=z^{-1-\nu-k} \times \\
(-1)^{l \nu+\kappa} \sum_{\sigma=0}^{\eta}\left(\begin{array}{l}
\eta \\
\sigma
\end{array}\right)\left(\log \left((-1)^{\kappa} z\right)\right)^{\eta-\sigma}\left(\left(-\frac{\partial}{\partial t}\right)^{\sigma}\left(R^{2+l}\right)\right)(\alpha, \nu+1+k, \nu) .
\end{gathered}
$$

Sine $u \pi / \sin (u \pi)$ is a even function, it follows from (11),(12), that

$$
\begin{gathered}
\operatorname{Res}\left(g_{l, \kappa}^{\sim} ;-1-\nu-k\right)=\frac{1}{(\kappa-3) !}(-1)^{l \nu+\kappa} z^{-1-\nu-k} \mathfrak{S}, \text { where } \mathfrak{S}= \\
\sum_{\theta \in[0,[(\kappa-3) / 2]] \cap \mathbb{Z}}\left(\begin{array}{c}
\kappa-3 \\
2 \theta
\end{array}\right)\left(\lim _{u \rightarrow 0}\left(\left(\frac{\partial}{\partial u}\right)^{2 \theta}\left(\frac{u \pi}{\sin (u \pi)}\right)^{\kappa-2}\right)\right) \mathfrak{S}^{*} \text { with } \mathfrak{S}^{*}= \\
\sum_{\sigma=0}^{\kappa-3-2 \theta}\left(\begin{array}{c}
\kappa-3-2 \theta \\
\sigma
\end{array}\right)\left(\log \left((-1)^{\kappa} z\right)\right)^{\kappa-3-2 \theta-\sigma}\left(\left(-\frac{\partial}{\partial t}\right)^{\sigma} R^{2+l}\right)(\alpha, \nu+1+k, \nu) .
\end{gathered}
$$

If $\kappa=3$ then $k=2$, and in view of (13),

$$
\begin{gathered}
\left.f_{\alpha, l, 2}^{*}(z, \nu):=f_{\alpha, l, 2}^{\vee *}(z, \nu):=\left((\nu+1)_{\alpha}\right)\right)^{2+l} f_{\alpha, l, 2}^{\vee}(z, \nu)= \\
\sum_{k=0}^{+\infty} z^{-(1+\nu+k)}\left((\nu+1)_{\alpha} R(\alpha, 1+\nu+k, \nu)\right)^{2+l},
\end{gathered}
$$


where $l=0,1,2$ and $\nu \in \mathbb{N}_{0}$. Let $t=1+\nu+k$ with $k \in[0,+\infty) \cap \mathbb{Z}$; then

$$
f_{\alpha, l, 2}^{*}(z, \nu)=\sum_{t=1+\nu}^{+\infty} z^{-t}\left((\nu+1)_{\alpha} R(\alpha, t, \nu)\right)^{2+l},
$$

where $l=0,1,2$ and $\nu \in \mathbb{N}_{0}$. If $\kappa=4$ then $k=3$ and $l \in\{0,1,2\}$. Let

$$
\begin{gathered}
f_{\alpha, l, 4}(z, \nu)^{\vee}:=f_{\alpha, l, 4}(z, \nu):=-\sum_{t=1+\nu}^{+\infty} z^{-t}\left(\frac{\partial}{\partial t}\left(R^{2+l}\right)\right)(\alpha, t, \nu), \\
f_{\alpha, l, k}^{\vee *}(z, \nu):=f_{\alpha, l, k}^{*}(z, \nu):=\left((\nu+1)_{\alpha}\right)^{2+l} f_{\alpha, l, k}(z, \nu),
\end{gathered}
$$

where $k=3,4, l=0,1,2$ and $\nu \in \mathbb{N}_{0}$. Then, in view of (13),

$$
f_{\alpha, l, 3}^{*}(z, \nu)=f_{\alpha, l, 2}^{*}(z, \nu) \log (z)+f_{\alpha, l, 4}^{*}(z, \nu) \text {. }
$$

If $\kappa=5$ then $k=5$ and $l \in\{1,2\}$. Let

$$
\begin{gathered}
f_{\alpha, l, 5}(z, \nu)=\frac{1}{2} \sum_{t=\nu+1}+\infty z^{-t} \sum_{k=0}^{2}\left(\left(\begin{array}{l}
2 \\
k
\end{array}\right)(-\log (z))^{2-k}\left(\frac{\partial}{\partial t}\right)^{k} R^{2+l}\right)(\alpha, t, \nu) \\
f_{\alpha, l, 6}(z, \nu)=2^{-1} \sum_{t=\nu+1}^{\infty} z^{-t}\left(\left(\frac{\partial}{\partial t}\right)^{2}\left(R^{2+l}\right)\right)(\alpha, t, \nu) \\
f_{\alpha, l, 5}^{\vee *}(z, \nu):=\left((\nu+1)_{\alpha}\right)^{2+l} f_{\alpha, l, 5}^{\vee}(z, \nu), f_{\alpha, l, k}^{*}(z, \nu):=\left((\nu+1)_{\alpha}\right)^{2+l} f_{\alpha, l, k}(z, \nu)
\end{gathered}
$$

for $k=5,6$. In view of (13),

$$
\begin{gathered}
f_{\alpha, l, 5}^{\vee}(z, \nu)= \\
i \pi \sum_{t=\nu+1}^{\infty}\left(\left(\sum_{k=0}^{1}(-\log (z))^{1-k}\left(\frac{\partial}{\partial t}\right)^{k}\right)(R)^{2+l}\right)(\alpha, t, \nu)+ \\
\frac{1}{2} \sum_{t=\nu+1}+\infty z^{-t} \sum_{k=0}^{2}\left(\left(\begin{array}{l}
2 \\
k
\end{array}\right)(-\log (z))^{2-k}\left(\frac{\partial}{\partial t}\right)^{k}(R)^{2+l}\right)(\alpha, t, \nu)= \\
-i \pi f_{\alpha, l, 3}^{\vee}(z, \nu)+f_{\alpha, l, 5}(z, \nu),
\end{gathered}
$$

and

$$
f_{\alpha, l, 5}(z, \nu)=\sum_{\sigma=0}^{2} \frac{1}{(2-\sigma) !}(\log (z))^{2-\sigma} f_{\alpha, l, 2+2 \sigma}^{\vee}(z, \nu)
$$


If $\kappa=6$ then $k=7$ and $l=2$. Let

$$
\begin{gathered}
f_{\alpha, l, 8}(z, \nu)=-6^{-1} \sum_{t=\nu+1}^{\infty} z^{-t}\left(\left(\frac{\partial}{\partial t}\right)^{3}\left(R^{2+l}\right)\right)(\alpha, t, \nu), \\
f_{\alpha, l, 7}(z, \nu)=\sum_{t=\nu+1}^{\infty} \frac{1}{6 z^{t}} \sum_{\sigma=0}^{3}\left(\begin{array}{l}
3 \\
\sigma
\end{array}\right)(\log (z))^{3-\sigma}\left(-\frac{\partial}{\partial t}\right)^{\sigma} R^{2+l}(\alpha, t, \nu)=(23) \\
\sum_{\text {sigma }=0}^{3} \frac{1}{(3-\sigma) !}(\log (z))^{3-\sigma} f_{\alpha, 2,2 \sigma+2}(z, \nu) . \\
f_{\alpha, l, 7}^{\vee *}(z, \nu):=\left((\nu+1)_{\alpha}\right)^{2+l} f_{\alpha, l, 7}^{\vee}(z, \nu), f_{\alpha, l, k}^{*}(z, \nu):=\left((\nu+1)_{\alpha}\right)^{2+l} f_{\alpha, l, k}(z, \nu)
\end{gathered}
$$

for $k=7,8$. In view of $(13)$,

$$
f_{\alpha, l, 7}^{\vee *}(z, \nu)=f_{\alpha, l, 7}^{*}(z, \nu)+\left(2 \pi^{2} / 3\right) f_{\alpha, l, 3}^{*}(z, \nu) .
$$

Since $(R(\alpha, t, \nu))^{2+l}$ for $\nu \in \mathbb{N}$ has in the points $t=1, \ldots, \nu$, the zeros of the order $2+l$, and $k-1<2+l$ for $k$ in $f_{\alpha \cdot l, 2 k}(z, \nu)$, it follows that

$$
\left.f_{\alpha . l, 2 k}(z, \nu)=\frac{(-1)^{k-1}}{(k-1) !} \sum_{t=1}^{\infty} z^{-t}\left(\frac{\partial}{\partial t}\right)^{k-1}\left((\nu+1)_{\alpha} R\right)^{2+l}\right)(\alpha, t, \nu)
$$

for $k \in[1.4] \cap \mathbb{Z}$ and $\nu \in \mathbb{N}_{0}$. For $m \in \mathbb{N}$ we denote by $E_{m}$ the unit $m \times m$ matrix. We denote by $\vec{e}_{m, k}$ and $\bar{e}_{m, k}$ respectively the $k$-th column and $k$-th row of the matrix $E_{m}$, if $k \in[1, m] \cap \mathbb{Z}$. We put $\vec{e}_{m, k}=0 \vec{e}_{m, 1}, \bar{e}_{m, k}=0 \bar{e}_{m, 1}$, if $k \in \mathbb{Z} \backslash[1, m]$. We denote by $N_{m}$ the $m \times m$-matrix, $k$-th column of which coincides with $\vec{e}_{m, k-1}$ for $k \in[1, m] \cap \mathbb{N}$. If $A$ is a matrix, then we denote by $A^{t}$ the matrix transposed to the matrix $A$. Let $\delta^{0} f_{\alpha, 2, k}(z, \nu)=f_{\alpha, 2, k}(z, \nu)$,

$$
X_{\alpha, 2, k}(z ; \nu)=\sum_{\kappa=1}^{8} \delta^{\kappa-1} f_{\alpha, 2, k}(z, \nu) \vec{e}_{8, k} \text { for } k \in \mathfrak{K} .
$$

The connection of $X_{\alpha, 2, k}(z ; \nu)$ with $X_{\alpha, 2, k}(z ; \nu-1)$ was studied in [7]. Related proofs are long and connected with heavy calculations. Therefore we decided to present here relatively easy case $l=2, \alpha=1$. We apply the same method, as in [4], where was considered the case $l=0, \alpha=1$; arithmetical applications of corresponding results can be found in [5] - [6]. Results for the case $\alpha=0$ can be found in [3]. Let $w$ be a independent variable,

$$
P(\alpha, w, \nu)=\nu^{7}(\nu+\alpha)^{4}+(2 \nu+\alpha) P^{(h)}(\alpha, w, \nu) \text { with } P^{(h)}(\alpha, w, \nu)=
$$




$$
\begin{aligned}
& \nu^{5}(\nu+\alpha)^{2}\left(-4 \nu(\nu+\alpha) w+2(8 \nu+5 \alpha) w^{2}\right)-4\left(11 \nu^{2}+15 \nu \alpha+5 \alpha^{2}\right) \times \\
& \nu^{4}(\nu+\alpha) w^{3}+\nu^{3}\left(96 \nu^{3}+210 \nu^{2} \alpha+150 \nu \alpha^{2}+35 \alpha^{3}\right) w^{4}-(3 \nu+2 \alpha) \times \\
& 4 \nu^{2}\left(15 \nu^{2}+21 \nu \alpha+7 \alpha^{2}\right) w^{5}+2 \nu\left(152 \nu^{3}+301 \nu^{2} \alpha+196 \nu \alpha^{2}+42 \alpha^{3}\right) w^{6}-4 w^{7} \times \\
& \left(119 \nu^{3}+228 \nu^{2} \alpha+144 \nu \alpha^{2}+30 \alpha^{3}\right), P^{*}(w, \nu)=\sum_{k=0}^{7} p_{k}^{*}(\nu) w^{k}=P(1, w, \nu), \\
& Q^{(h)}(\alpha, w, \nu)=\nu^{3}\left(704 \nu^{3}+1314 \nu^{2} \alpha+810 \nu \alpha^{2}+165 \alpha^{3}\right)+4 w \nu^{2} \times \\
& \left(455 \nu^{3}+859 \nu^{2} \alpha+535 \nu \alpha^{2}+110 \alpha^{3}\right)+2 w^{2} \nu \times \\
& \left(800 \nu^{3}+1523 \nu^{2} \alpha+956 \nu \alpha^{2}+198 \alpha^{3}\right)+4 w^{3} \times \\
& \left(119 \nu^{3}+228 \nu^{2} \alpha+144 \nu \alpha^{2}+30 \alpha^{3}\right), Q(\alpha, w, \nu)=(2 \nu+\alpha) Q^{(h)}(\alpha, w, \nu), \\
& Q^{*}(\alpha, w, \nu)=Q(\alpha, w+1, \nu)(w-\nu-\alpha)^{4}, Q(w, \nu)=Q(1, w, \nu), Q^{*}(w, \nu)= \\
& \sum_{k=0}^{7} q_{k}^{*}(\nu) w^{k}=Q^{*}(1, w, \nu), T^{*}(z, w, \nu)=P^{*}(w, \nu)+z Q^{*}(w, \nu) \text {. Let } \\
& \tau=\tau_{1}(\nu)=\nu+1, \mu=\mu_{1}(\nu)=(\nu+1)^{2}=\tau^{2}, \kappa^{2}=\kappa, \mathfrak{k} \in[0,3] \cap \mathbb{Z}, \\
& r_{2 \mathfrak{k}+2-\kappa}^{*}(\nu)=\kappa\left(\begin{array}{l}
4 \\
\mathfrak{k}
\end{array}\right)\left(-\tau^{2}\right)^{4-\mathfrak{k}}, \bar{R}(\nu)=\sum_{k=1}^{8} r_{k}^{*}(\nu) \bar{e}_{8, k}, h_{i, k}^{*}(\nu)=p_{i-1}^{*}(\nu) r_{k}^{*}(\nu), \\
& S^{*}(\nu)=p_{0}^{*}(\nu) E_{8}+\sum_{k=1}^{7} p_{k}^{*}(\nu) N_{8}^{k} \\
& v_{i, k}^{*}(\nu)=\left(\sum_{\sigma=1}^{k}\left(\begin{array}{c}
i-1 \\
k-\sigma
\end{array}\right) q_{\sigma-1}^{*}(\nu)\right)+\sum_{\sigma=1}^{k} \sum_{\kappa=1}^{i-1} h_{9-\kappa, \sigma}^{*}(\nu)\left(\begin{array}{c}
i-1-\kappa \\
k-\sigma
\end{array}\right) .
\end{aligned}
$$

where $\{i, k\} \subset[1,8] \cap \mathbb{Z}$. Let $V^{*}(\nu)$ be the $8 \times 8$-matrix which has $v_{i, k}^{*}(\nu)$ on intersection of its $i$-th row and $k$-th column. Let further

$$
A^{*}(z, \nu)=S^{*}(\nu)+z V^{*}(\nu), X_{k}(z ; \nu)=X_{1,2, k}(z ; \nu) .
$$

We prove here the following

Theorem ??.1. The domain of definition of column $X_{k}(z ; \nu)$ can be extended to the set $\Omega_{0} \times(\mathbb{Z} \backslash\{-1\})$, and this column satisfies to the equation

$$
\nu^{11} X_{k}(z ; \nu-1)=A^{*}(z ; \nu) X_{k}(z ; \nu)
$$

for $\left.\nu \in M_{1}^{*}=(-\infty,-2] \cup[1,+\infty)\right) \cap \mathbb{Z}, k=1,2,3,5,7, z \in \Omega_{0}$. 


\section{1 Some Relations. Begin of the Proof of Theorem ??.1}

Let $\alpha$ be an independent variable.

Lemma 1.1. $\operatorname{Let}^{\wedge}(\alpha, w, \nu):=(w+\nu)^{4} P(\alpha, w, \nu)+w^{8} Q(\alpha, w, \nu)-\nu^{11}(w-$ $\nu-\alpha)^{4}$. Then

$$
T^{\wedge}(\alpha, w, \nu)=0
$$

Proof. If $T^{\wedge}(\alpha, w, \nu) \neq 0$, then $\operatorname{deg}_{\alpha}\left(T^{\wedge}(\alpha, w, \nu)\right) \leq 4$. Moreover, coefficient at $\alpha^{4}$ in $T^{\wedge}(\alpha, w, \nu)$ is equal to

$$
\begin{gathered}
\left(\nu^{7}-4 \nu^{6} w+10 \nu^{5} w^{2}-20 \nu^{4} w^{3}+35 \nu^{3} w^{4}-56 \nu^{2} w^{5}+84 \nu w^{6}-120 w^{7}\right) \times \\
(w+\nu)^{4}+w^{8}\left(165 \nu^{3}+440 \nu^{2} w+396 \nu w^{2}+120 w^{3}\right)-\nu^{11}= \\
\left(\nu^{11}(1-1)+(4-4) \nu^{10}\right) w+2(3-8+5) \nu^{9} w^{2}+4(1-6+10-5) \nu^{8} w^{3}+ \\
(1-16+60-80+35) \nu^{7} w^{4}-4(1-10+30-35+14) \nu^{6} w^{5}+12(7-40+33) \nu w^{10}+ \\
(10-80+210-224+84) \nu^{5} w^{6}-4(5-35+84-84+30) \nu^{4} w^{7}+ \\
(35-224+504-480+165) \nu^{3} w^{8}-8(7-42+90-55) \nu^{2} w^{9}-120(1-1) w^{11}=0 .
\end{gathered}
$$

Hence, $\operatorname{deg}_{\alpha}\left(T^{\wedge}(\alpha, w, \nu)\right) \leq 3$, if $T^{\wedge}(\alpha, w, \nu) \neq 0$, and it is enough to check the equality (34) for $\alpha=-2 \nu, \alpha=-\nu, \alpha=0$, and $\alpha=\nu$. If $\alpha=-2 \nu$, then

$$
P(-2 \nu, w, \nu)=\nu^{11}, Q(-2 \nu, w, \nu)=0,(w-\nu-(-2 \nu))^{4}=(w+\nu)^{4},
$$

and (34) holds. If $\alpha=-\nu$, then $P(-\nu, w, \nu)=\nu^{7} w^{4}-4 \nu^{6} w^{5}+10 \nu^{5} w^{6}-$

$$
\begin{gathered}
20 \nu^{4} w^{7}, Q(-\nu, w, \nu)=35 \nu^{7}+84 \nu^{6} w+70 \nu^{5} w^{2}+20 \nu^{4}, T^{\wedge}(-\nu, w, \nu)= \\
\left(\nu^{7} w^{4}-4 \nu^{6} w^{5}+10 \nu^{5} w^{6}-20 \nu^{4} w^{7}\right)(w+\nu)^{4}-\nu^{11} w^{4}+ \\
w^{8}\left(35 \nu^{7}+84 \nu^{6} w+70 \nu^{5} w^{2}+20 \nu^{11}\right)=20(-1+1) \nu^{4} w^{11}+ \\
(1-1) \nu^{11} w^{4}+(-4+4) \nu^{10} w^{5}+(10-16+6) \nu^{9} w^{6}+(-20+40-24+4) \nu^{8} w^{7}+ \\
(-80+60-16+1+35) \nu^{7} w^{8}+(-120+40-4+84) \nu^{6} w^{9}+10(-8+1+7) \nu^{5} w^{10}=0 . \\
\text { If } \alpha=0, \text { then } P(0, w, \nu)=\nu^{11}-8 \nu^{10} w+32 \nu^{9} w^{2}-88 \nu^{8} w^{3}+ \\
8\left(24 \nu^{7} w^{4}-45 \nu^{6} w^{5}+76 \nu^{5} w^{6}-119 \nu^{4} w^{7}\right), Q(0, w, \nu)=8 \times \\
\left(176 \nu^{7}+455 \nu^{6} w+400 \nu^{5} w^{2}+119 \nu^{4} w^{3}, T^{\wedge}(0, w, \nu)=(w+\nu)^{4} \times\right. \\
\left(\nu^{11}-8 \nu^{10} w+32 \nu^{9} w^{2}-88 \nu^{8} w^{3}+192 \nu^{7} w^{4}-360 n u^{6} w^{5}+608 \nu^{5} w^{6}-952 \nu^{4} w^{7}\right)+ \\
w^{8}\left(1408 \nu^{7}+3640 \nu^{6} w+3200 \nu^{5} w^{2}+952 \nu^{4} w^{3}\right)-\nu^{11}(w-\nu)^{4}=
\end{gathered}
$$




$$
\begin{aligned}
& \nu^{4} w^{11} 952(-1+1)+\nu^{15}(1-1)+\nu^{14} w(4-8+4)+\nu^{13} w^{2}(32-32+6-6)+ \\
& \nu^{12} w^{3}(-88+128-48+4+4)+\nu^{11} w^{4}(192-352+192-32+1-1)+ \\
& \nu^{10} w^{5}(-360+768-528+128-8)+\nu^{9} w^{6} 32(19-45+36-11+1)+ \\
& \nu^{8} w^{7}(-952+2432-2160+768-88)+\nu^{7} w^{8} 32(-119+114-45+6+44)+ \\
& \nu^{6} w^{9} 8(-714+304-45+455)+\nu^{5} w^{10} 32(-119+19+100)=0 . \\
& \text { If } \alpha=\nu \text {, then } P(\nu, w, \nu)=16 \nu^{11}-96 \nu^{10} w+312 \nu^{9} w^{2}-744 \nu^{8} w^{3}+ \\
& 1473 \nu^{7} w^{4}-2580 \nu^{6} w^{5}+4146 \nu^{5} w^{6}-6252 \nu^{4} w^{7}, Q(\nu, w, \nu)= \\
& 3 \nu^{4}\left(2993 \nu^{3}+5877 \nu^{2} w+6954 \nu w^{2}+2084 w^{3}\right), T_{2}^{\wedge}(\nu, w, \nu)= \\
& (w+\nu)^{4}\left(16 \nu^{11}-96 \nu^{10} w+312 \nu^{9} w^{2}-744 \nu^{8} w^{3}\right)+ \\
& (w+\nu)^{4}\left(1473 \nu^{7} w^{4}-2580 \nu^{6} w^{5}+4146 \nu^{5} w^{6}-6252 \nu^{4} w^{7}\right)+ \\
& w^{8} 3 \nu^{4}\left(2993 \nu^{3}+5877 \nu^{2} w+6954 \nu w^{2}+2084 w^{3}\right)-\nu^{11}(w-2 \nu)^{4}= \\
& 16(1-1) \nu^{1} 5+32(-3+2+1) \nu^{14} w+24(13-16+4-1) \nu^{13} w^{2}+ \\
& 8(-93+156-72+8+1) \nu^{12} w^{3}+(16-384+1872-2976+1473-1) \nu^{11} w^{4}+ \\
& 12(-8+104-372+491-215) \nu^{10} w^{5}+6(52-496+1473-1720+691) \nu^{9} w^{6}+ \\
& 12(-62+491-1290+1382-521) \nu^{8} w^{7}+ \\
& 3(491-3440+8292-8336+2993) \nu^{7} w^{8}+12(-215+1382-3126+1959) \nu^{6} w^{9}+ \\
& 6(691-4168+3477) \nu^{5} w^{10}+6252(-1+1) \nu^{4} w^{11}=0 .
\end{aligned}
$$

Let $\nu \in \mathfrak{M}_{0}=\mathbb{Z} \backslash\{-1\}, \tau=\nu+1$

$$
D(z, \nu, w)=z\left(w^{2}-\tau^{2}\right)^{4}-w^{8} .
$$

and $w$ is independent variable. Clearly,

$$
D(z, \nu, w)=D(z,-\nu-2, w) \text { for } \nu \in \mathbb{Z}
$$

Let $\delta:=z \frac{\partial}{\partial z}$. According to the general properties of the Mejer's functions,

$$
D(z, \nu, \delta) f=0,(\delta+\nu+1)^{4} f_{k}^{\vee}(z, \nu)=(\delta-\nu-2)^{4} f_{k}^{\vee}(z, \nu+1)
$$

where $f=f_{k}^{\vee}(z, \nu), \nu \in \mathbb{N}_{0}, k \in \mathfrak{K}=\{1,2,3,5,7\}$. Therefore

$$
(\delta+\nu)^{4} f_{k}^{\vee}(z, \nu-1)=(\delta-\nu-1)^{4} f_{k}^{\vee}(z, \nu),
$$


where $\nu \in \cap \mathbb{N}, k \in \mathfrak{K}$. Let

$$
f_{k}^{\vee}(z,-\nu-2)=f_{k}^{\vee}(z, \nu), \text { for } \nu \in \mathbb{N}_{0}, k \in \mathfrak{K} .
$$

So, $f_{k}^{\vee}(z, \nu)$ is defined for $\nu \in \mathfrak{M}_{0}$. If $\nu \in(-\infty,-2] \cap \mathbb{Z}$, then $\nu_{1}=-\nu-2 \in \mathbb{N}_{0}$, and, in view of $(39), f_{k}^{\vee}(z, \nu)=f_{k}^{\vee}\left(z,-\nu_{1}-2\right)=f_{k}^{\vee}\left(z, \nu_{1}\right)=f_{k}^{\vee}(z,-\nu-2)$. Therefore (39) holds for all the $\nu \in \mathfrak{M}_{0}$. Furthermore,

$$
\delta^{s} f_{k}^{\vee}(z,-\nu-2)=\delta^{s} f_{k}^{\vee}(z, \nu) \text { for } s \in \mathbb{N}_{0}, \nu \in \mathfrak{M}_{0}, k \in \mathfrak{K} .
$$

In view of (36) and (40), the first equality in (37) holds for $\nu \in \mathfrak{M}_{0}$. Furthermore, if $\nu \in(-\infty,-2] \cap \mathbb{Z}$, then $\nu_{1}=-\nu-2 \in \mathbb{N}_{0},-\nu-1 \in \mathbb{N}$, and in view of $(37)$, (40),

$$
\begin{gathered}
(\delta-\nu-1)^{4} f_{k}^{\vee}(z, \nu)=\left(\delta+\nu_{1}+1\right)^{4} f_{k}^{\vee}\left(z, \nu_{1}\right)= \\
\left(\delta-\nu_{1}-2\right)^{4} f_{k}^{\vee}\left(z, \nu_{1}+1\right)=(\delta+\nu)^{4} f_{k}^{\vee}(z,-\nu-1)=(\delta+\nu)^{4} f_{k}^{\vee}(z, \nu-1) .
\end{gathered}
$$

If $\nu \in(-\infty,-3] \cap \mathbb{Z}$, then $\nu+1 \in(-\infty,-2] \cap \mathbb{Z}$, and, in view (41), we have the equality $(\delta-\nu-2)^{4} f_{k}^{\vee}(z, \nu+1)=(\delta+\nu+1)^{4} f_{k}^{\vee}(z, \nu)$. So, (38) holds for $\nu \in \mathfrak{M}_{1}=((-\infty,-2] \cap \mathbb{Z}) \cup \mathbb{N}$, and second equality in (37) holds for $\nu \in$ $\mathfrak{M}_{2}=((-\infty,-3] \cap \mathbb{Z}) \cup \mathbb{N}_{0}$. In view of (34), (38), (41), (35),(37) and equalities $Q(\delta, \nu)(\delta+\nu)^{4}=(\delta+\nu)^{4} Q(\delta, \nu), Q(\delta, \nu) z=z Q(\delta+1, \nu)$,

$$
\begin{gathered}
\nu^{11}(\delta+\nu)^{4} f_{k}^{\vee}(z, \nu-1)=(\delta+\nu)^{4} P^{*}(\delta, \nu) f_{k}^{\vee}(z, \nu)+ \\
\delta^{8} Q(\delta, \nu) f_{k}^{\vee}(z, \nu)=(\delta+\nu)^{4}\left(P(\delta, \nu)+z Q^{*}(\delta, \nu)\right) f_{k}^{\vee}(z, \nu) .
\end{gathered}
$$

Clearly, $\mathfrak{M}_{i}=((-\infty,-1-i] \cup[2-i,+\infty)) \cap \mathbb{Z}=\mathfrak{M}_{0} \backslash\{2-2 i\}$, for $i \in\{1,2\}$, and the map $\nu \rightarrow \nu_{1}=-\nu-2$ is bijection of $\mathfrak{M}_{i}$ onto $\mathfrak{M}_{3-i}$ for $i \in\{1,2\}$. We denote by $\mathfrak{V}_{i}$ the set of all functions defined on $\Omega_{0} \times \mathfrak{M}_{i}$ for $i=0,1,2$ and regular relatively $z \in \Omega_{0}$ for fixed $\nu$. For $f \in \mathfrak{V}_{0}, z \in \Omega_{0}$, we put

$$
\begin{gathered}
\mathfrak{W}_{1}(f, z, \nu)=\nu^{11} f(z, \nu-1)-T^{*}(z, \delta, \nu) f(z, \nu) \text { if } \nu \in \mathfrak{M}_{1}, \\
\mathfrak{W}_{2}(f, z, \nu)=\mathfrak{W}_{1}(f, z,-\nu-2) \text { if } \nu \in \mathfrak{M}_{2} .
\end{gathered}
$$

The map $f \rightarrow \mathfrak{W}_{i}(f, z, \nu)$ turns $\mathfrak{V}_{0}$ into $\mathfrak{V}_{i}$ for $i=1,2$. In view of (42),

$$
(\delta+\nu)^{4} \mathfrak{W}_{1}\left(f_{k}^{\vee}, z, \nu\right)=0 \text { for } \nu \in \mathfrak{M}_{1}, k \in \mathfrak{K} .
$$

If $\nu \in \mathfrak{M}_{2}, k \in \mathfrak{K}$, then $-\nu-3 \in \mathfrak{M}_{2}, \nu_{1}=-\nu-2 \in \mathfrak{M}_{1}$, and, in view of (45), (40),

$$
(\delta-\nu-2)^{4} \mathfrak{W}_{2}\left(f_{k}^{\vee}, z, \nu\right)=0 .
$$


Theorem 1.1. Instead of (45) and (46) the following equality holds

$$
\mathfrak{W}_{i}\left(f_{k}^{\vee}, z, \nu\right)=0 \text {. }
$$

where $i \in\{1,2\}, \nu \in \mathfrak{M}_{i}, k \in \mathfrak{K}$. In other words, this means that $(\delta+\nu)^{4}$ and $(\delta-\nu-2)^{4}$ can be omit from the left side of respectively (45) and (46).

Proof. Let $\theta=2 \nu+1, i \in \mathbb{N}$.

$$
\begin{gathered}
S_{i}(t, \nu)=\left(\frac{\partial}{\partial t}\right)^{i-1}\left(\left(\sum_{k=1}^{\nu} \frac{1}{t-k}\right)-\sum_{k=0}^{\nu+1} \frac{1}{t+k}\right)=(-1)^{i-1}(i-1) ! \times \quad(48) \\
\left(\left(\sum_{k=1}^{\nu} \frac{1}{(t-k)^{i}}\right)-\sum_{k=0}^{\nu+1} \frac{1}{(t+k)^{i}}\right), H_{i}^{\wedge}(\nu)=S_{i}(\nu, \nu-1)-S_{i}(\nu+1, \nu)= \\
(-1)^{i}(i-1) !\left(2 / \nu^{i}-1 /(2 \nu+1)^{i}-1 /(2 \nu+2)^{i}\right), H_{i}^{\vee}(\nu)=H_{i}^{\wedge}(\nu) \times \\
(2 \nu(2 \nu+1)(2 \nu+2))^{i}=(-1)^{i}(i-1) !\left(2^{i+1}(\theta+1)^{i} \theta^{i}-(\theta-1)^{i}\left(\theta^{i}+(\theta+1)^{i}\right)\right)= \\
(-1)^{i}(i-1) !\left(\left(2^{i+1}(\theta+1)^{i}-(\theta-1)^{i}\right) \theta^{i}-\left(\theta^{2}-1\right)^{i}\right) . \text { Then } \\
H_{1}^{\vee}(\nu)=-2 \theta^{2}-5 \theta-1, H_{2}^{\vee}(\nu)=6 \theta^{4}+18 \theta^{3}+9 \theta^{2}-1,\left(H_{1}^{\vee}(\nu)\right)^{2}=4 \theta^{4}+ \\
+29 \theta^{2}+10 \theta+1,4\left(H_{1}^{\vee}(\nu)\right)^{2}+3 H_{2}^{\vee}(\nu)=34 \theta^{4}+134 \theta^{3}+143 \theta^{2}+40 \theta+1 \\
4\left(H_{1}^{\vee}(\nu)\right)^{3}+3 H_{1}^{\vee}(\nu) H_{2}^{\vee}(\nu)=-68 \theta^{6}-438 \theta^{5}-990 \theta^{4}-929 \theta^{3}- \\
345 \theta^{2}-45 \theta-1, H_{3}^{\vee}(\nu)=-28 \theta^{6}-102 \theta^{5}-96 \theta^{4}-34 \theta^{3}+6 \theta^{2}-2, \\
\left.\left.(R(t, \nu))^{4}\left(64 S_{1}^{3}(t, \nu)\right)+48 S_{1}(t, \nu)\right) S_{2}(t, \nu)+4 S_{3}(t, \nu)\right) . \\
\left.\left(\frac{\partial}{\partial t}\right)(R(t, \nu))^{4}=(R(t, \nu))^{4} 4 S_{1}(t, \nu),\left(\frac{\partial}{\partial t}\right)^{2}(R(t, \nu))^{4}\right)= \\
S_{i}^{2}\left(\nu, \nu-300 \theta^{6}-1854 \theta^{5}-4056 \theta^{4}-3750 \theta^{3}-1374 \theta^{2}-180 \theta-6\right. \\
\left(H_{1}^{\vee}(\nu)\right)^{3}=-8 \theta^{6}-60 \theta^{5}-162 \theta^{4}-185 \theta^{3}-81 \theta^{2}-15 \theta-1, H_{i}^{\wedge \wedge}(\nu):=
\end{gathered}
$$


If the function $f(z)$ is regular in $\Omega_{0}$, then, according to the Leibnitz formula, we have the equality

$$
\begin{gathered}
\delta^{n}\left(\frac{(\log (z))^{m}}{m !} f(z)\right)=\left.\sum_{k=0}^{m} \frac{\left((\log (z))^{m-k}\right.}{(m-k) !}\left(\begin{array}{l}
n \\
k
\end{array}\right) w^{n-k}\right|_{w=\delta} f(z)= \\
\left(\left.\sum_{k=0}^{m} \frac{(\log (z))^{m-k}}{(m-k) ! k !}\left(\left(\frac{\partial}{\partial w}\right)^{k} w^{n}\right)\right|_{w=\delta}\right) f(z) ;
\end{gathered}
$$

therefore, if $H(w) \in \mathbb{C}[w]$, then

$$
H(\delta)\left(\frac{(\log (z))^{m}}{m !} f(z)\right)=\left.\sum_{k=0}^{m} \frac{(\log (z))^{m-k}}{(m-k) ! k !}\left(\left(\frac{\partial}{\partial w}\right)^{k} H(w)\right)\right|_{w=\delta} f(z) .
$$

If $f \in \mathfrak{V}_{0}$, then $\left((\log (z))^{m} / m !\right) f(z) \in \mathfrak{V}_{0}$ for any $m \in \mathbb{N}$, and

$$
\begin{gathered}
\mathfrak{W}_{i}\left(\left(\left(\log ^{m}(z)\right) / m !\right) f, z, \nu\right)=\left(\left(\log ^{m}(z)\right) / m !\right) \mathfrak{W}_{i}(f, z, \nu)- \\
\sum_{k=1}^{m} \frac{(\log (z))^{m-k}}{(m-k) ! k !}\left(\left.\left(\frac{\partial}{\partial w}\right)^{k} T^{*}\left(z, w, \eta_{i}(\nu)\right)\right|_{w=\delta} f\left(z, \eta_{i}(\nu)\right)\right.
\end{gathered}
$$

with $\eta_{i}(\nu)=(3-2 i) \nu+2-2 i$ for $i=1,2$. In view of $(2),(43), \mathfrak{W}_{1}^{\vee}\left(f_{1}, z, \nu\right)$ belongs to $\mathbb{C}[z]$ for arbitrary $\nu \in \mathfrak{M}_{1}$. In view of $(45), \mathfrak{W}_{1}\left(f_{1}, z, \nu\right)$ belongs to the null-space of the operator $(\delta+\nu)^{4}$ (as linear operator on $\mathbb{C}[z]$ ), and in the case, when $\nu \in \mathbb{N}$, this null-space consits of the single element - the zero element in $\mathbb{C}[z]$. Hence, $\mathfrak{W}_{1}\left(f_{1}^{\vee}, z, \nu\right)=0$ for $\nu \in \mathbb{N}$. In view of (28),

$$
Q(-\nu, \nu)=\nu^{3}(2 \nu+1)^{4}, Q(\nu+2,-\nu-2)=-(\nu+2)^{3}(2 \nu+3)^{4} .
$$

In view of (2), (44), $\mathfrak{W}_{2}\left(f_{1}, z, \nu\right) \in \mathbb{C}[z]$ if $\nu \in \mathfrak{M}_{2}$. In view of (46), the null-space of the operator $(\delta-\nu-2)^{4}$ (as linear operator on $\mathbb{C}[z]$ ) contains $\mathfrak{W}_{2}\left(f_{1}, z, \nu\right)$, and, if $\nu \in \mathbb{N}_{0}$, then $\mathfrak{W}_{2}\left(f_{1}, z, \nu\right)=\gamma_{1} z^{\nu+2}$, with $\gamma_{1} \in \mathbb{C}$.

Remark 1. Of course, the null-space of the operator $(\delta-\nu-2)^{4}$ as linear operator on $\mathbb{C}[z, \log (z)]$, consists of all the functions having the form

$$
z^{\nu+2}\left(\beta_{0}+\beta_{1} \log (z)+\beta_{2} \log ^{2}(z)+\beta_{3} \log ^{3}(z)\right) \text { with } \beta_{k} \in \mathbb{C} \text { for } k=0,1,2,3 .
$$

But in our case the space $\mathbb{C}[z]$ is an invariant subspace of the space $\mathbb{C}[z, \log (z)]$ relatively this operator. Therefore $\beta_{k}=0$ for $k=1,2,3$.

Clearly,

$$
p(\delta) z^{k}=p(k) z^{k} \text { for any } p \in \mathbb{C}, k \in \mathbb{Z} .
$$


In view of $(2), \operatorname{deg}_{z}\left(f_{1}^{\vee}(z, \nu+\kappa)=\nu+1+\kappa\right.$, coefficient at $z^{\nu+1+\kappa}$ in $f_{1}^{\vee}(z, \nu+\kappa)$ is equal to $((2 \nu+2 \kappa+1) !)^{4} /((\nu+\kappa+1) !)^{8}$, for $\kappa \in\{0,1\}$.

$$
\begin{gathered}
\text { In view of } \left.\left.(54) \text { and (53), } z Q(\delta+1,-\nu-2)(\delta+\nu+1)^{4}\right)\right) z^{\nu+1}= \\
Q(\nu+2,-\nu-2)(2 \nu+2)^{4} z^{\nu+2}=-(\nu+2)^{3}(2 \nu+3)^{4}(2 \nu+2)^{4} z^{\nu+2} . \\
\text { In view of } \left.(44),(\nu+1) !)^{8} \gamma_{1} /(2 \nu+1) !\right)^{4}= \\
-(\nu+2)^{11}(2 \nu+3)^{4}(2 \nu+2)^{4} /(\nu+2)^{8}-\left(-(\nu+2)^{3}(2 \nu+3)^{4}(2 \nu+2)^{4}\right)=0 .
\end{gathered}
$$

Therefore (47) holds for $i=2, k=1, \nu \in \mathbb{N}_{0}$.

We denote by $\mathfrak{C}$ the set of all the functions $f(z)$, which are regular outside the closed unit circle and which tend to zero, when $z$ tends to $\infty$. Each such function is the sum of convergent for $|z|>1$ Laurent series $\sum_{k=1}^{\infty} a_{k} z^{-k}$.

We denote by $\mathfrak{C}^{(n)}$ with $n \in \mathbb{N}_{0}$ the set of all the functions $f(z)$, can be represented in the form

$$
f(z)=\sum_{k=0}^{n} f_{k}(z) \frac{(\log (z))^{k}}{k !}
$$

with $f_{k}(z) \in \mathfrak{C}$ for $k=0, \ldots, n$. Clearly,

$$
(\delta f)(z)=\left(\delta f_{n}\right)(z) \frac{(\log (z))^{n}}{n !}+\sum_{k=0}^{n-1}\left(\left(\delta f_{k}\right)(z)+f_{k+1}(z)\right) \frac{(\log (z))^{k}}{k !}
$$

Clearly, if $\{m, n, \nu\} \subset \mathbb{N}_{0}$ and $m<n$, then $\mathfrak{C}^{(m)}$ and $z^{-\nu} \mathfrak{C}^{(n)}$ are $\delta$ invariant $\mathbb{C}$-linear subspaces of $\mathbb{C}$-linear space $\mathfrak{C}^{(n)}$. Let $\mathbb{C}[[x]]$ denotes the ring of all formal power series over $\mathbb{C}$ with variable $x$. Let $n \in \mathbb{N}_{0}$; we consider elements of $\oplus_{k=0}^{n} \mathbb{C}\left[\left[z^{-1}\right]\right]=\mathbb{C}\left[\left[z^{-1}\right]\right] \overbrace{\oplus, \quad \ldots, \oplus \mathbb{C}}^{n+1 \text { times }}\left[\left[z^{-1}\right]\right]$ as columns

$$
\Theta(z)=\sum_{k=0}^{n} T_{k}(z) \vec{e}_{n+1, k+1}
$$

consisting of $n+1$ elements $T_{0}(z), \ldots, T_{n}(z)$, which lie in $\mathbb{C}\left[\left[z^{-1}\right]\right]$. We put

$$
\delta T(z)=\sum_{k=0}^{\infty}-k a_{k} z^{-k} \text { for } T(z)=\sum_{k=0}^{\infty} a_{k} z^{-k} \in \mathbb{C}\left[\left[z^{-1}\right]\right]
$$


If $\Theta(z) \in \oplus_{k=0}^{n} \mathbb{C}\left[\left[z^{-1}\right]\right]$ represented in the form (57), we put

$$
(\delta \Theta)(z)=\sum_{k=0}^{n} T_{k}^{(1)}(z) \vec{e}_{n+1, k+1}
$$

where $T_{n}^{(1)}(z)=\left(\delta T_{n}\right)(z)$ and $T_{k}^{(1)}(z)=\left(\delta T_{k}\right)(z)+T_{k+1}(z)$, if $0 \leq k<n$.

Lemma 1.1. If $n \in \mathbb{N}_{0}$ then $\mathfrak{C}^{(n)}$ can be considered as (or maybe, more precisely, embedded in) a $\delta$-invariant $\mathbb{C}$-linear subspace of $\oplus_{k=0}^{n} \mathbb{C}\left[\left[z^{-1}\right]\right]$.

Proof. We need to prove only that if

$$
f(z)=\sum_{k=0}^{n} f_{k}(z) \frac{(\log (z))^{k}}{k !}=0, \text { where } f_{k}(z) \in \mathfrak{C}
$$

for $k=0, \ldots, n$, then $f_{k}(z)=0$ for $k=0, \ldots, n$. The equality $(60)$ can be prolonged by means of analytic continuation to the equality

$$
\begin{gathered}
H((r, \varphi))=\sum_{k=0}^{n} f_{k}\left(\exp (\log (Z)) \frac{(\log (Z))^{k}}{k !}=\right. \\
\sum_{k=0}^{n} f_{k}(\exp (\ln (r)+i \varphi)) \frac{(\ln (r)+i \varphi)^{k}}{k !}=\sum_{k=0}^{n} f_{k}(r \exp (i \varphi)) \frac{(\ln (r)+i \varphi)^{k}}{k !}=0,
\end{gathered}
$$

where $Z=(r, \phi)$ lies on Rimannian surface of $\log (Z), r>1, \varphi \in \mathbb{R}$ and $H$ is uniquely defined by $h$.

$$
\text { Then } H((r, \varphi+2 \kappa \pi))=\sum_{k=0}^{n} f_{k}(r \exp (i \varphi)) \frac{\left(\ln (r)+i(\varphi+2 \kappa \pi)^{k}\right.}{k !}=0
$$

for $\kappa=0, \ldots, n$. Making use of Vandermonde determinant we conclude that $f_{k}(r \exp (i \varphi))=0$ for $k=0, \ldots, n$.

In view of $(15),(44),(13),(21),(20),(23), \mathfrak{W}_{2}\left(f_{k}, z, \nu\right)$ belongs to $z^{-\nu} \mathfrak{C}^{(3)}$ for $\nu \in \mathbb{N}_{0}$. If $\left\{\nu \in \mathbb{N}_{0}\right.$, then $(\delta-\nu-2)^{2+l}$ as operator on linear over $\mathbb{C}$ space $z^{-\nu} \mathfrak{C}^{(0)}$ is injection. Therefore, in view of $(46), \mathfrak{W}_{2}\left(f_{k}^{\vee}, z, \nu\right)=0$ for $k \in\{2,3,5,7\}, \nu \in \mathbb{N}_{0}$. Let $\nu \in \mathbb{N}$. In view of $(7)$, if $\kappa=\kappa^{2}$, then

$$
\left(R((\nu+1-\kappa, \nu-\kappa))^{4}=((\nu-\kappa) !)^{8} /((2 \nu+2-2 \kappa) !)^{4} .\right.
$$

In view of $(45), \mathfrak{W}_{1}\left(f_{2}, z, \nu\right)$ belongs to the null-space of the operator $(\delta+\nu)^{4}$ as linear operator on $z^{-\nu} \mathfrak{C}^{(0)}$, and, if $\nu \in \mathbb{N}$, then $\mathfrak{W}_{1}\left(f_{2}, z, \nu\right)=\gamma_{2} z^{-\nu}$. 
Remark 2. The null-space of $(\delta+\nu)^{4}$ as linear operator on $\mathfrak{C}[\log (z)]$ coincides with $z^{-\nu} \mathfrak{C}^{(3)}$. But in our case the space $z^{-\nu} \mathfrak{C}^{(0)}=z^{-\nu} \mathfrak{C}$ is an invariant subspace of the space $z^{-\nu} \mathfrak{C}[\log (z)]$ relatively this operator. Therefore $\mathfrak{W}_{2}\left(f_{k}, z, \nu\right) \in z^{-\nu \mathfrak{C}^{(0)}}$ for $\nu \in \mathbb{N}_{0}$.

In view of $(54,(14),(7)$, and (53),

$$
\begin{gathered}
\gamma_{2}=-\nu^{11}((\nu-1) !)^{8} /((2 \nu) !)^{4}+Q(-\nu, \nu)(-2 \nu-2)^{4}(\nu !)^{8} /((2 \nu+2) !)^{4}= \\
-\nu^{11}((\nu-1) !)^{8} /((2 \nu) !)^{4}+\nu^{3}(2 \nu+1)^{4}(2 \nu+2)^{4}(\nu !)^{8} /((2 \nu+2) !)^{4}=0 .
\end{gathered}
$$

Hence, $\mathfrak{W}_{1}\left(f_{2}^{\vee}, z, \nu\right)=0$ for $\nu \in \mathbb{N}$. Therefore, in view of (45), (13) and (52),

$$
\begin{gathered}
\mathfrak{W}_{1}\left(f_{3}, z, \nu\right)=\mathfrak{W}_{1}\left((\log (z)) f_{2}, z, \nu\right)+\mathfrak{W}_{1}\left(f_{4}, z, \nu\right)=\mathfrak{W}_{1}\left(f_{4}, z, \nu\right)- \\
\left.\left(\frac{\partial}{\partial w} T^{*}(z, w, \nu)\right)\right|_{w=\delta} f_{2}(z, \nu)=\nu^{11} f_{4}^{\vee}(z, \nu-1)-T^{*}(z, \delta, \nu) f_{4}^{\vee}(z, \nu)- \\
\left.\left(\frac{\partial}{\partial w} T^{*}(z, w, \nu)\right)\right|_{w=\delta} f_{2}(z, \nu) \in \mathbb{C}\left[\left[z^{-1}\right]\right] .
\end{gathered}
$$

We deduce from (28) by means of standard calculations the following equalities

$$
\begin{gathered}
\frac{Q(-\nu, \nu)}{(1+2 \nu)^{4} \nu^{3}}=1,\left.\left(\frac{d}{d w} \frac{Q(w, \nu)}{4(1+2 \nu)^{3} \nu^{2}}\right)\right|_{w=-\nu}=2+3 \nu \\
\left.\left(\left(\frac{d}{d w}\right)^{2} \frac{Q(w, \nu)}{4 \nu^{3}(1+2 \nu)^{4}}\right)\right|_{w=-\nu}=\frac{3}{(1+2 \nu)^{2}}+\frac{18}{\nu^{2}}-\frac{16}{\nu(1+2 \nu)} \\
\left(\frac{d}{d w}\right)^{3} \frac{Q(w, \nu)}{48 \nu+24}=\left(119 \nu^{3}+228 \nu^{2}+144 \nu+30\right) .
\end{gathered}
$$

In view (53), (64) and (48), if $\kappa^{2}=\kappa$, then

$$
\left.\left(\frac{d}{d w} \frac{Q^{*}(w-\kappa, \nu)}{4 \nu^{3}(1+2 \nu)^{4}(2+2 \nu)^{4}}\right)\right|_{w=-\nu-1+\kappa}=-H_{1}^{\wedge}(\nu) .
$$

In view of $(45),(63), \mathfrak{W}_{1}\left(f_{3}, z, \nu\right)$, for $\nu \in \mathbb{N}$, belongs to the null-space of the operator $(\delta+\nu)^{4}$ on the $\delta$-invariant linear subspace $z^{-\nu} \mathfrak{C}^{(0)}$ of $z^{-\nu} \mathfrak{C}^{(1)}$; hence $\mathfrak{W}_{1}\left(f_{3}, z, \nu\right)=\gamma_{3} z^{-\nu}$ with $\gamma_{3} \in \mathbb{C}$. In view of (16), (48), (50), (62), (65),

$$
\begin{gathered}
\gamma_{3} z^{-\nu}=-\left.\nu^{11} \frac{\partial}{\partial t}(R(t, \nu-1))^{4}\right|_{t=\nu} z^{-\nu}-\left.\frac{\partial\left(-(R(t, \nu))^{4}\right)}{\partial t}\right|_{t=\nu+1} \times \\
z Q^{*}(\delta, \nu) z^{-\nu-1}-\left.(R(\nu+1, \nu))^{4} z \frac{\partial\left(Q^{*}(w, \nu)\right)}{\partial w}\right|_{w=\delta} z^{-\nu-1}=
\end{gathered}
$$




$$
\left.4 \nu^{3}(\nu !)^{8} z^{-\nu}\left(-S_{1}(\nu, \nu-1)+S_{1}(\nu+1, \nu)+H_{1}^{\wedge}(\nu)\right) /(2 \nu) !\right)^{4}=0 .
$$

Therefore $\mathfrak{W}_{1}\left(f_{3}^{\vee}, z, \nu\right)=0$ for $\nu \in \mathbb{N}$. So, (47) holds for $k \in\{2,3\}, \nu \in \mathbb{N}$. In view of (43), (52), (18), (21), (63),

$$
\begin{gathered}
\mathfrak{W}_{1}\left(f_{5}, z, \nu\right)=\sum_{k=0}^{2} \mathfrak{W}_{1}\left((\log (z))^{k} f_{6-2 k}, z, \nu\right) / k != \\
h_{5,0}(z)+\log (z) h_{5,1}(z), \text { with } \\
h_{5,1}(z)=\mathfrak{W}_{1}\left(f_{4}, z, \nu\right)-\left.\frac{\partial T^{*}(z, w, \nu)}{\partial w}\right|_{w=\delta} f_{2}(z, \nu)=\mathfrak{W}_{1}\left(f_{3}, z, \nu\right)=0, \\
h_{5,0}(z)=\mathfrak{W}_{1}\left(f_{6}, z, \nu\right)-\left.\sum_{k-1}^{2} \frac{1}{2}\left(\left(\frac{\partial}{\partial w}\right)^{k} \frac{T^{*}(z, w, \nu)}{k !}\right)\right|_{w=\delta} f_{6-2 k}(z, \nu) .
\end{gathered}
$$

In view of (69) and (70), $\mathfrak{W}_{1}\left(f_{5}, z, \nu\right) z^{-\nu} \in \mathfrak{C}^{(0)}$. Hence, in view of (45), $\mathfrak{W}_{1}\left(f_{5}, z, \nu\right)$ belongs to the null-space of the operator $(\delta+\nu)^{4}$ on the $\delta$-invariant linear subspace $z^{-\nu} \mathfrak{C}^{(0)}$ of the space $z^{-\nu} \mathfrak{C}^{(2)}$ and, if $\nu \in \mathbb{N}$ then $\mathfrak{W}_{1}\left(f_{5}, z, \nu\right)=$ $\gamma_{5} z^{-\nu}$ with $\gamma_{5} \in \mathbb{C}$. In view of (50), (62), (48), (19), (53), (43), coefficient at $z^{-\nu}$ in $\mathfrak{W}_{1}\left(f_{6}, z, \nu\right)$ is equal to

$$
\begin{gathered}
\left(\nu^{3}(\nu !)^{8}\left(8\left(\left(S_{1}(\nu, \nu-1)\right)^{2}-\left(S_{1}(\nu+1, \nu)\right)^{2}\right)+2 H_{2}^{\vee}(\nu)\right)\right) /((2 \nu) !)^{4}= \\
\left.\left(\nu^{3}(\nu !)^{8}\left(8 H_{1}^{\wedge}(\nu)\left(2 S_{1}(\nu+1, \nu)+H_{1}^{\wedge}(\nu)\right)+2 H_{2}^{\wedge}(\nu)\right)\right) /((2 \nu) !)^{4}\right)= \\
\nu^{3}(\nu !)^{8}\left(16 H_{1}^{\wedge}(\nu) S_{1}(\nu+1, \nu)+8\left(H_{1}^{\wedge}(\nu)\right)^{2}+2 H_{2}^{\wedge}(\nu)\right) /((2 \nu) !)^{4} .
\end{gathered}
$$

In view of (67), (50), (62) and (48),

$$
\begin{aligned}
& \text { coefficient at } z^{-\nu} \text { in }-\left.z\left(\frac{\partial}{\partial w} Q^{*}(w, \nu)\right)\right|_{w=\delta} f_{4}(z, \nu) \text { is equal to } \\
& -4 \nu^{3}(1+2 \nu)^{4}(2 \nu+2)^{4} H_{1}^{\vee}(\nu)\left(4(\nu !)^{8} S_{1}(\nu+1, \nu)\right) /((2 \nu+2) !)^{4} .
\end{aligned}
$$

In view of (53), (65), (67), if $\kappa^{2}=\kappa$, then

$$
\begin{gathered}
\left.\frac{1}{4 \nu^{3}(1+2 \nu)^{4}(2+2 \nu)^{4}}\left(\frac{d}{d w}\right)^{2} Q^{*}(w-\kappa, \nu)\right|_{w=-\nu-1+\kappa}= \\
\left(3 /(1+2 \nu)^{2}+18 / \nu^{2}-16 / \nu(1+2 \nu)\right)-8(2+3 \nu) / \nu(1+2 \nu)(2+2 \nu)+ \\
3 /(2+2 \nu)^{2}=3 /(2+2 \nu)^{2}+3 /(1+2 \nu)^{2}+18 / \nu^{2}-16 / \nu(1+2 \nu)- \\
16 / \nu(2+2 \nu)+8 /(2 \nu+1)(2+2 \nu)=\left(4\left(H_{1}^{\wedge}(\nu)\right)^{2}+H_{2}^{\wedge}(\nu)\right) .
\end{gathered}
$$


In view of (62), (73), coefficient at $z^{-\nu}$ in $\left.\frac{1}{2}\left(\left(\frac{\partial}{\partial w}\right)^{2} Q^{*}(w, \nu)\right)\right|_{w=\delta} f_{2}(z, \nu)$ is equal to $2 \nu^{3}(\nu !)^{8}((2 \nu) !)^{-4}\left(4\left(H_{1}^{\wedge}(\nu)\right)^{2}+H_{2}^{\wedge}(\nu)\right)$; in view of $(71),(72),(69)$, we have $\gamma_{5}=0$. So, (47) holds for $k \in\{1,2,3,5\}, \nu \in \mathbb{N}$. Let $k=7, \nu \in \mathbb{N}$. Since (47) holds for $k=2$ it follows from (43), (52), (18), (23), that

$$
\begin{gathered}
\mathfrak{W}_{1}\left(f_{7}, z, \nu\right)=\sum_{k-0}^{3}\left(\left(\left(\log ^{k}(z)\right) / k !\right) f_{8-2 k}, z, \nu\right)= \\
h_{7,0}(z)+(\log (z)) h_{7,1}(z)+\left(\left(\log ^{2}(z)\right) / 2\right) h_{7,2}(z),
\end{gathered}
$$

where, in view of $(63),(70)$,

$$
\begin{gathered}
h_{7,3-i}(z)=-\left.\sum_{k=1}^{i}\left(\left(\frac{\partial}{\partial w}\right)^{k} \frac{T^{*}(z, w, \nu)}{k !}\right)\right|_{w=\delta} f_{2+2 i-2 k}(z, \nu)+ \\
\mathfrak{W}_{1}\left(f_{2+2 i}, z, \nu\right)=h_{5,2-i}=0 \text { for } i=1,2 . \\
h_{7,0}(z)=\mathfrak{W}_{1}\left(f_{8}, z, \nu\right)-\left.\sum_{k=1}^{3}\left(\left(\frac{\partial}{\partial w}\right)^{k} \frac{T^{*}(z, w, \nu)}{k !}\right)\right|_{w=\delta} f_{8-2 k}(z, \nu) .
\end{gathered}
$$

In view of $(74),(75), \mathfrak{W}_{1}\left(f_{7}, z, \nu\right) \in \mathfrak{C}^{(0)}$. in view of $(45), \mathfrak{W}_{1}\left(f_{7}, z, \nu\right)$ belongs to the null-space of the operator $(\delta+\nu)^{4}$ on the $\delta$-invariant linear subspace $z^{-\nu} \mathfrak{C}^{(0)}$ of the space $z^{-\nu} \mathfrak{C}^{(3)}$. Consequently, $\mathfrak{W}_{1}\left(f_{7}, z, \nu\right)=\gamma_{7} z^{-\nu}$ with $\gamma_{7} \in \mathbb{C}$.

We conclude from (50), (62), (48), (22), (53) and (43) that coefficient at $z^{-\nu}$ in $-3((2 \nu) !)^{4} \mathfrak{W}_{1}\left(f_{8}, z, \nu\right) /\left(2 \nu^{3}(\nu !)^{8}\right)$ is equal to

$$
\begin{gathered}
16\left(\left(S_{1}(\nu, \nu-1)\right)^{3}-\left(S_{1}(\nu+1, \nu)\right)^{3}\right)+ \\
12\left(S_{1}(\nu, \nu-1) S_{2}(\nu, \nu-1)-S_{1}(\nu+1, \nu) S_{2}(\nu+1, \nu)\right)+H_{3}^{\wedge}(\nu)= \\
\left.\left.16\left(3 H_{1}^{\wedge}(\nu) S_{1}^{2}(\nu+1, \nu)+3\left(H_{1}^{\wedge}\right)(\nu)\right)^{2} S_{1}(\nu+1, \nu)+\left(H_{1}^{\wedge}\right)(\nu)\right)^{3}\right)+ \\
12\left(H_{1}^{\wedge}(\nu) S_{2}(\nu+1, \nu)+H_{1}^{\wedge}(\nu) H_{2}^{\wedge}(\nu)+S_{1}(\nu+1, \nu) H_{2}^{\wedge}(\nu)\right)+H_{3}^{\wedge}(\nu),
\end{gathered}
$$

In view of (54), (67) and (50), coefficient at $z^{-\nu}$ in

$$
\begin{gathered}
-3((2 \nu) !)^{4}\left(\left.\frac{\partial}{\partial w} T^{*}(z, w, \nu)\right|_{w=\delta} \frac{f_{6}(z, \nu)}{\left(2 \nu^{3}(\nu !)^{8}\right)}\right. \text { is equal to } \\
12 H_{1}^{\wedge}(\nu)\left(4 S_{1}^{2}(\nu+1, \nu)+S_{2}(\nu+1, \nu)\right) . \\
\text { Hence, }-3(2 \nu) !)^{4}\left(\mathfrak{W}_{1}\left(f_{8}, z, \nu\right)-\left.\left(\frac{\partial}{\partial w} T^{*}(z, w, \nu)\right)\right|_{w=\delta} f_{6}(z, \nu)\right) /\left(2 \nu^{3}(\nu !)^{8}\right)
\end{gathered}
$$


has coefficient at $z^{-\nu}$ equal to result of subtraction (78) from (77), i.e.

$$
\begin{gathered}
\left.\left.16\left(3\left(H_{1}^{\wedge}\right)(\nu)\right)^{2} S_{1}(\nu+1, \nu)+\left(H_{1}^{\wedge}\right)(\nu)\right)^{3}\right)+ \\
12\left(H_{1}^{\wedge}(\nu) H_{2}^{\wedge}(\nu)+S_{1}(\nu+1, \nu) H_{2}^{\wedge}(\nu)\right)+H_{3}^{\wedge}(\nu),
\end{gathered}
$$

In view of (54), (73), (50), (62), (48), coefficient at $z^{-\nu}$ in

$$
\begin{gathered}
-\left.3((2 \nu) !)^{4}\left(\left(\frac{\partial}{\partial w}\right)^{2} T^{*}(z, w, \nu)\right)\right|_{w=\delta} \frac{f_{4}(z, \nu)}{\left(4 \nu^{3}(\nu !)^{8}\right)} \text { is equal to } \\
\left(48\left(H_{1}^{\wedge}(\nu)\right)^{2}+12 H_{2}^{\wedge}(\nu)\right) S_{1}(\nu+1, \nu) .
\end{gathered}
$$

In view of $(79),(80),(73)$, coefficient at $z^{-\nu}$ in

$$
\mathfrak{W}_{1}\left(f_{8}, z, \nu\right)-\left.\sum_{k=1}^{2}\left(\left(\frac{\partial}{\partial w}\right)^{k} \frac{T^{*}(z, w, \nu)}{k !}\right)\right|_{w=\delta} f_{8-2 k}(z, \nu)
$$

is equal to

$$
\left.\left.-\nu^{3}(\nu !)^{8}\left(32\left(H_{1}^{\wedge}(\nu)\right)^{3}+24 H_{1}^{\wedge}(\nu) H_{2}^{\wedge}(\nu)\right)+2 H_{3}^{\wedge}(\nu)\right) /(3(2 \nu) !)^{4}\right) .
$$

In view of (66) and (53),

$$
\begin{gathered}
\left.\left(\left(\frac{d}{d w}\right)^{3} \frac{8 Q^{*}(w, \nu)}{6(2 \nu+1)(2 \nu+2)}\right)\right|_{w=-\nu-1}= \\
\left.\left(\left(\frac{d}{d w}\right)^{3} \frac{8 Q(w, \nu)(w-\nu-2)^{4}}{6(2 \nu+1)(2 \nu+2)}\right)\right|_{w=-\nu}=\left(119 \nu^{3}+228 \nu^{2}+144 \nu+30\right) \times \\
32(2 \nu+2)^{3}-64 \nu(2 \nu+1)(2 \nu+2)^{2}\left(18+56 \nu+43 \nu^{2}\right)+192 \nu^{2}(2 \nu+1)^{2} \times \\
(2 \nu+2)(2+3 \nu)-32 \nu^{3}(2 \nu+1)^{3}=4(\theta+1)^{3} \times \\
\left(119(\theta-1)^{3}+456(\theta-1)^{2}+576(\theta-1)+240\right)-8(\theta-1) \theta(\theta+1)^{2} \times \\
\left.476(\theta-1)^{2}+112(\theta-1)+72\right)+24(\theta-1)^{2} \theta^{2}(\theta+1)(3 \theta+1)-4(\theta-1)^{3} \theta^{3}= \\
344\left(\theta^{2}-\theta\right)\left(\theta^{2}-1\right)^{2}-896 \theta\left(\theta^{2}-1\right)^{2}-576\left(\theta^{2}-\theta\right)(\theta+1)^{2}+ \\
24\left(\theta^{4}-\theta^{2}\right)\left(3 \theta^{2}-2 \theta-1\right)-4(\theta-1)^{3} \theta^{3}=(476-344+72-4) \theta^{6}+ \\
(1824+344-896-48+12) \theta^{5}+(-1428+1824+2304+688-96-12-576) \theta^{4}+
\end{gathered}
$$




$$
\begin{gathered}
(-3648+4608+960-688+1792-576+48+4) \theta^{3}+ \\
(1428-3648+2880-344+576+24) \theta^{2}+ \\
(1824-4608+2880+344-896+576) \theta+(-476+1824-2304+960)= \\
200 \theta^{6}+1236 \theta^{5}+2704 \theta^{4}+2500 \theta^{3}+1374 \theta^{2}+120 \theta+4= \\
-2\left(16\left(H_{1}^{\vee}(\nu)\right)^{3}+12 H_{1}^{\vee}(\nu) H_{2}^{\vee}(\nu)+H_{3}^{\vee}(\nu)\right) / 3 .
\end{gathered}
$$

Hence, coefficient at $z^{-\nu}$ in

$$
\left.\left(\left(\frac{\partial}{\partial w}\right)^{3} \frac{T^{*}(z, w, \nu)}{3 !}\right)\right|_{w=\delta} f_{2}(z, \nu)
$$

is equal to

$$
\begin{gathered}
\left.-((2 \nu+1)(2 \nu+2) / 8)\left((\nu !)^{8} /((2 \nu+2) !)^{4}\right)\right) \times \\
2\left(16\left(H_{1}^{\vee}(\nu)\right)^{3}+12 H_{1}^{\vee}(\nu) H_{2}^{\vee}(\nu)+H_{3}^{\vee}(\nu)\right) / 3= \\
\left.-\left((\nu)^{3}(\nu !)^{8}\right) /(3(2 \nu) !)^{4}\right)\left(32\left(H_{1}^{\wedge}(\nu)\right)^{3}+24 H_{1}^{\wedge}(\nu) H_{2}^{\wedge}(\nu)+2 H_{3}^{\wedge}(\nu)\right) .
\end{gathered}
$$

In view of $(76),(81),(83), \gamma_{7}=0$.

So, (47) holds for $i \in\{1,2\}, k \in\{1,2,3,5,7\}, \nu \in(2-i,+\infty) \cap \mathbb{Z}_{0}$. If $i=1,2 \nu \in(-\infty,-1-i] \cap \mathbb{Z}$, then $\nu_{1}=-\nu-2 \in[i-1,+\infty) \cap \mathbb{Z}$, and $\mathfrak{W}_{i}\left(f_{k}^{\vee}, z, \nu\right)=\mathfrak{W}_{3-i}\left(f_{k}^{\vee}, z, \nu_{1}\right)=0$. So, (47) holds for $k \in\{1,2,3,5,7\}$.

\section{2 Solution of an Auxiliary Difference Equation}

Let $j \in[1,9] \cap \mathbb{N}, \bar{y}_{j}^{*}(\nu)=\sum_{k=1}^{8} y_{j, k}^{*}(\nu) \bar{e}_{8, k}$ is the solution of difference equation

$$
\bar{y}_{j+1}^{*}(\nu)=\bar{y}_{j}^{*}(\nu)\left(E_{8}+N_{8}\right)+p_{8-j}^{*}(\nu) \bar{R}(\nu)
$$

with $j \in[1,8] \cap \mathbb{N}$, with $\bar{R}(\nu)$ defined in (29) and with initial values

$$
\bar{y}_{1}^{*}(\nu)=\bar{v}_{1}^{*}(z ; \nu)=\sum_{\eta=0}^{7} q_{\eta}^{*}(\nu) \bar{e}_{8, \eta+1}
$$

Lemma 2.1 Let $j \in[1,8] \cap \mathbb{Z}, k \in[0,9-j] \cap \mathbb{Z}$. Then

$$
\bar{y}_{j+k}^{*}(\nu)=\bar{y}_{j}^{*}(\nu)\left(E_{8}+N_{8}\right)^{k}+\bar{R}(\nu) \sum_{\kappa=1}^{k} p_{9-j-\kappa}^{*}(\nu)\left(E_{8}+N_{8}\right)^{k-\kappa} .
$$


Proof. For $k=0$ the equality (86) is evident, for $k=1$ it follows from (84). Let $1 \leq k \leq 8-j$ and (86) holds for this $k$. Then $j+k \in[1,8] \cap \mathbb{Z}$, and we apply (84), for $j+k$ instead $j$. Then we have

$$
\begin{gathered}
\bar{y}_{j+k+1}^{*}(\nu)=\bar{y}_{j+k}^{*}(\nu)\left(E_{8}+N_{8}\right)+p_{8-j-k}^{*}(\nu) \bar{R}(\nu)=\bar{y}_{j}^{*}(\nu)\left(E_{8}+N_{8}\right)^{k+1}+ \\
\bar{R}(\nu) \sum_{\kappa=1}^{k} p_{9-j-\kappa}^{*}(\nu)\left(E_{8}+N_{8}\right)^{k+1-\kappa}+p_{8-j-k}^{*}(\nu) \bar{R}(\nu)\left(E_{8}+N_{8}\right)^{0}= \\
\left.\bar{y}_{j}^{*}(\nu)\left(E_{8}\right)+N_{8}\right)^{k+1}+\bar{R}(\nu)\left(\sum_{\kappa=1}^{k+1} p_{\alpha, 2,9-j-\kappa}^{*}(\nu)\left(E_{8}+N_{8}\right)^{k+1-\kappa},\right) .
\end{gathered}
$$

Hence (86) holds for all $k \in[1,9-i] \cap \mathbb{Z}$. $\square$ If we take in (86) $j=1, i=j+k$, and apply $(85)$ then we see that $\bar{y}_{i}^{*}(\nu)$ s equal to

$$
\left(\sum_{\sigma=1}^{8} q_{\sigma-1}^{*}(\nu) \bar{e}_{8, \sigma}\left(E_{8}+N_{8}\right)^{i-1}\right)+\bar{R}(\nu) \sum_{\kappa=1}^{i-1} p_{8-\kappa}^{*}(\nu)\left(E_{8}+N_{8}\right)^{i-1-\kappa} .
$$

Therefore, in view of $(31)$,

$$
y_{i, k}^{*}(\nu)=v_{i, k}^{*}(\nu) \text { for } i \in[1,8] \cap \mathbb{Z}, k \in[1,8] \cap \mathbb{Z} .
$$

In view of $(85), y_{1, j}^{*}(\nu)=q_{j-1}^{*}(\nu)$ for $j \in[1,8] \cap \mathbb{Z}$. In view of $(84),(29)$,

$$
y_{i+1, j}^{*}(\nu)=y_{i, j}^{*}(\nu)+y_{i, j-1}^{*}(\nu)+p_{8-i}^{*}(\nu) r_{k}^{*}(\nu) \text { if }\{i, j\} \subset[1,8] \cap \mathbb{Z} \text {. }
$$

In view of (27), (29),

$$
\begin{gathered}
p_{0}^{*}(\nu)=(\tau-1)^{7} \tau^{4}=\tau^{11}-7 \tau^{10}+21 \tau^{9}-35 \tau^{8}+35 \tau^{7}-21 \tau^{6}+ \\
7 \tau^{5}-\tau^{4}, p_{1}^{*}(\nu)=-4(2 \tau-1)(\tau-1)^{6} \tau^{3}=-8 \tau^{10}+52 \tau^{9}-144 \tau^{8}+ \\
220 \tau^{7}-200 \tau^{6}+108 \tau^{5}-32 \tau^{4}+4 \tau^{3}, p_{2}^{*}(\nu)=2 \tau^{2}(\tau-1)^{5}(2 \tau-1) \times \\
(8 \tau-3)=32 \tau^{9}-188 \tau^{8}+466 \tau^{7}-630 \tau^{6}+500 \tau^{5}-232 \tau^{4}+58 \tau^{3}- \\
6 \tau^{2}, p_{3}^{*}(\nu)=-4(\tau-1)^{4}(2 \tau-1) \tau\left(11 \tau^{2}-7 \tau+1\right)=-88 \tau^{8}+452 \tau^{7}- \\
964 \tau^{6}+1100 \tau^{5}-720 \tau^{4}+268 \tau^{3}-52 \tau^{2}+4 \tau, p_{4}^{*}(\nu)=(2 \tau-1)(\tau-1)^{3} \times \\
\left(96 \tau^{3}-78 \tau^{2}+18 \tau-1\right)=192 \tau^{7}-828 \tau^{6}+1446 \tau^{5}-1310 \tau^{4}+655 \tau^{3}- \\
177 \tau^{2}+23 \tau-1, p_{5}^{*}(\nu)=-4(\tau-1)^{2}(2 \tau-1)(3 \tau-1)\left(15 \tau^{2}-9 \tau+1\right)= \\
-4\left(90 \tau^{6}-309 \tau^{5}+414 \tau^{4}-275 \tau^{3}+95 \tau^{2}-16 \tau+1\right), p_{6}^{*}(\nu)=2(\tau-1) \times
\end{gathered}
$$




$$
\begin{gathered}
(2 \tau-1)\left(152 \tau^{3}-155 \tau^{2}+50 \tau-5\right)=2\left(304 \tau^{5}-766 \tau^{4}+717 \tau^{3}-315 \tau^{2}\right)+ \\
130 \tau-10, p_{7}^{*}(\nu)=-4(2 \tau-1)\left(119 \tau^{3}-129 \tau^{2}+45 \tau-5\right)=-952 \tau^{4}+ \\
4\left(377 \tau^{3}-219 \tau^{2}+55 \tau-5\right) .
\end{gathered}
$$

In view of $(28), Q^{(h)}(1, w+1, \nu)=704 \nu^{6}+3134 \nu^{5}+5846 \nu^{4}+5827 \nu^{3}+$

$$
\begin{gathered}
3264 \nu^{2}+972 \nu+120+4\left(455 \nu^{5}+1659 \nu^{4}+2415 \nu^{3}+1750 \nu^{2}+630 \nu+90\right) w+ \\
2\left(800 \nu^{4}+2237 \nu^{3}+2324 \nu^{2}+1062 \nu+180\right) w^{2}+4\left(119 \nu^{3}+228 \nu^{2}+144 \nu+30\right) w^{3}= \\
4\left(119 \tau^{3}-129 \tau^{2}+45 \tau-5\right) w^{3}+2\left(800 \tau^{4}-963 \tau^{3}+413 \tau^{2}-75 \tau+5\right) w^{2}+ \\
4\left(455 \tau^{5}-616 \tau^{4}+329 \tau^{3}-91 \tau^{2}+14 \tau-1\right) w+704 \tau^{6}-1090 \tau^{5}+736 \tau^{4}- \\
297 \tau^{3}+79 \tau^{2}-13 \tau+1, Q(w, \nu)=1408 \tau^{7}-2884 \tau^{6}+2562 \tau^{5}-1330 \tau^{4}+
\end{gathered}
$$$$
455 \tau^{3}-105 \tau^{2}+15 \tau-1+4\left(910 \tau^{6}-1687 \tau^{5}+1274 \tau^{4}-511 \tau^{3}+119 \tau^{2}-16 \tau+1\right) w+
$$$$
2\left(1600 \tau^{5}-2726 \tau^{4}+1789 \tau^{3}-563 \tau^{2}+85 \tau-5\right) w^{2}+952 \tau^{4} w^{3}+
$$$$
4\left(-377 \tau^{3}+219 \tau^{2}-55 \tau+5\right) w^{3}=\sum_{k=0}^{3} q_{k}(\nu) w^{k} \text {. In view of (85), (89), }
$$

$$
\begin{gathered}
y_{1,1}^{*}(\nu)=q_{0}^{*}(\nu)=\tau^{4} q_{0}(\nu)=1408 \tau^{11}-2884 \tau^{10}+2562 \tau^{9}-1330 \tau^{8}+ \\
\left.455 \tau^{7}-105 \tau^{6}+15 \tau^{5}-\tau^{4}, y_{1,2}^{*}(\nu)=q_{1}^{*}(\nu)=-4 \tau^{3} q_{0}(\nu)+\tau^{4} q_{1}^{(} \nu\right)= \\
4\left(-498 \tau^{10}+1197 \tau^{9}-1288 \tau^{8}+819 \tau^{7}-336 \tau^{6}+89 \tau^{5}-14 \tau^{4}+\tau^{3}\right), \\
y_{1,3}^{*}(\nu)=6 \tau^{2} q_{0}-4 \tau^{3} q_{1}(\nu)+\tau^{4} q_{2}(\nu)=-6 \tau^{2}+74 \tau^{3}-384 \tau^{4}+ \\
2\left(498 \tau^{5}-465 \tau^{6}-717 \tau^{7}+2118 \tau^{8}+2118 \tau^{8}-1456 \tau^{9}\right), y_{1,4}^{*}(\nu)= \\
-4 \tau q_{0}(\nu)+6 \tau^{2} q_{1}(\nu)-4 \tau^{3} q_{2}(\nu)+\tau^{4} q_{3}(\nu)=4 \tau-36 \tau^{2}+76 \tau^{3}+ \\
4\left(94 \tau^{4}-665 \tau^{5}+1723 \tau^{6}-2163 \tau^{7}+1090 \tau^{8}\right), y_{1,5}^{*}(\nu)=q_{0}(\nu)-4 \times \\
\tau q_{1}(\nu)+6 \tau^{2} q_{2}(\nu)-4 \tau^{3} q_{3}(\nu)+\tau^{4} q_{4}(\nu)=-1-\tau+91 \tau^{2}-509 \tau^{3}+ \\
970 \tau^{4}+142 \tau^{5}-2572 \tau^{6}+2240 \tau^{7}, y_{1,6}^{*}(\nu)=q_{1}(\nu)-4 \tau q_{2}(\nu)+6 \tau^{2} q_{3}(\nu)= \\
4\left(1-6 \tau-21 \tau^{2}+285 \tau^{3}-990 \tau^{4}+1503 \tau^{5}-862 \tau^{6}\right), y_{1,7}^{*}(\nu)=q_{2}(\nu)-4 \tau q_{3}(\nu) \\
=-10+90 \tau-246 \tau^{2}+74 \tau^{3}+580 \tau^{4}-608 \tau^{5}, y_{1,8}^{*}(\nu)=q_{3}(\nu)=-p_{7}^{*}(\nu) .
\end{gathered}
$$

Consecutive calculations according to (88),(29) give the following results:

$$
y_{2,1}^{*}(\nu)=-952 \tau^{12}+2916 \tau^{11}-3760 \tau^{10}+2782 \tau^{9}-1350 \tau^{8}+455 \tau^{7}-
$$




$$
\begin{aligned}
& 105 \tau^{6}+15 \tau^{5}-\tau^{4}, y_{2,2}^{*}(\nu)=1408 \tau^{11}-4876 \tau^{10}+7350 \tau^{9}-6482 \tau^{8}+ \\
& 3731 \tau^{7}-1449 \tau^{6}+371 \tau^{5}-57 \tau^{4}+4 \tau^{3}, y_{2,3}^{*}(\nu)=-6 \tau^{2}+78 \tau^{3}-440 \tau^{4}+8 \\
& 1352 \tau^{5}-2194 \tau^{6}+962 \tau^{7}+2588 \tau^{8}-4156 \tau^{9}+1816 \tau^{10}, y_{2,4}^{*}(\nu)=-2912 \tau^{9}+ \\
& 8596 \tau^{8}-10086 \tau^{7}+5962 \tau^{6}-1664 \tau^{5}-8 \tau^{4}+150 \tau^{3}-42 \tau^{2}+4 \tau, y_{2,5}^{*}(\nu)= \\
& -1352 \tau^{8}+2636 \tau^{7}-936 \tau^{6}-1198 \tau^{5}+1226 \tau^{4}-433 \tau^{3}+55 \tau^{2}+3 \tau-1, \\
& y_{2,6}^{*}(\nu)=2240 \tau^{7}-6020 \tau^{6}+6154 \tau^{5}-2990 \tau^{4}+631 \tau^{3}+7 \tau^{2}-25 \tau+3, \\
& y_{2,7}^{*}(\nu)=360 \tau^{6}-628 \tau^{5}+124 \tau^{4}+334 \tau^{3}-250 \tau^{2}+66 \tau-6, y_{2,8}^{*}(\nu)=-p_{6}^{*}(\nu) \text {, } \\
& y_{3,1}^{*}(\nu)=608 \tau^{13}-2484 \tau^{12}+4350 \tau^{11}-4390 \tau^{10}+2912 \tau^{9}-1360 \tau^{8}+455 \tau^{7}- \\
& 105 \tau^{6}+15 \tau^{5}-\tau^{4}, y_{3,2}^{*}(\nu)=-952 \tau^{12}+4324 \tau^{11}-8636 \tau^{10}+10132 \tau^{9}- \\
& 7832 \tau^{8}+4186 \tau^{7}-1554 \tau^{6}+386 \tau^{5}-58 \tau^{4}+4 \tau^{3}, y_{3,3}^{*}(\nu)=-1024 \tau^{11}+ \\
& 3068 \tau^{10}-2542 \tau^{9}-1374 \tau^{8}+4173 \tau^{7}-3603 \tau^{6}+1723 \tau^{5}-497 \tau^{4}+82 \tau^{3}- \\
& 6 \tau^{2}, y_{3,4}^{*}(\nu)=1816 \tau^{10}-7068 \tau^{9}+11184 \tau^{8}-9124 \tau^{7}+3768 \tau^{6}-312 \tau^{5}- \\
& 448 \tau^{4}+228 \tau^{3}-48 \tau^{2}+4 \tau, y_{3,5}^{*}(\nu)=736 \tau^{9}-1948 \tau^{8}+1154 \tau^{7}+1246 \tau^{6}- \\
& 2082 \tau^{5}+1158 \tau^{4}-283 \tau^{3}+13 \tau^{2}+7 \tau-1, y_{3,6}^{*}(\nu)=-1352 \tau^{8}+4876 \tau^{7}- \\
& 6956 \tau^{6}+4956 \tau^{5}-1764 \tau^{4}+198 \tau^{3}+62 \tau^{2}-22 \tau+2, y_{3,7}^{*}(\nu)=192 \tau^{7}+ \\
& 468 \tau^{6}-210 \tau^{5}-346 \tau^{4}+445 \tau^{3}-203 \tau^{2}+41 \tau-3, y_{3,8}^{*}(\nu)=-p_{5}^{*}(\nu), \\
& y_{4,1}^{*}(\nu)=-360 \tau^{14}+1844 \tau^{13}-4140 \tau^{12}+5450 \tau^{11}-4770 \tau^{10}+2976 \tau^{9}- \\
& 1364 \tau^{8}+455 \tau^{7}-105 \tau^{6}+15 \tau^{5}-\tau^{4}, y_{4,2}^{*}(\nu)=608 \tau^{13}-3436 \tau^{12}+ \\
& 8674 \tau^{11}-13026 \tau^{10}+13044 \tau^{9}-9192 \tau^{8}+4641 \tau^{7}-1659 \tau^{6}+401 \tau^{5}- \\
& 59 \tau^{4}+4 \tau^{3}, y_{4,3}^{*}(\nu)=488 \tau^{12}-1644 \tau^{11}+1056 \tau^{10}+3190 \tau^{9}-7686 \tau^{8}+ \\
& 8103 \tau^{7}-5141 \tau^{6}+2109 \tau^{5}-555 \tau^{4}+86 \tau^{3}-6 \tau^{2}, y_{4,4}^{*}(\nu)=-1024 \tau^{11}+ \\
& 4884 \tau^{10}-9610 \tau^{9}+9810 \tau^{8}-4951 \tau^{7}+165 \tau^{6}+1411 \tau^{5}-945 \tau^{4}+310 \tau^{3}- \\
& 54 \tau^{2}+4 \tau, y_{4,5}^{*}(\nu)=-344 \tau^{10}+1084 \tau^{9}-700 \tau^{8}-1370 \tau^{7}+2734 \tau^{6}-2010 \tau^{5}+ \\
& 686 \tau^{4}-55 \tau^{3}-35 \tau^{2}+11 \tau-1, y_{4,6}^{*}(\nu)=736 \tau^{9}-3300 \tau^{8}+6030 \tau^{7}- \\
& 5710 \tau^{6}+2874 \tau^{5}-606 \tau^{4}-85 \tau^{3}+75 \tau^{2}-15 \tau+1, y_{4,7}^{*}(\nu)=88 \tau^{8}-260 \tau^{7}+ \\
& 136 \tau^{6}+346 \tau^{5}-590 \tau^{4}+387 \tau^{3}-125 \tau^{2}+19 \tau-1, y_{4,8}^{*}(\nu)=-p_{4}^{*}(\nu),
\end{aligned}
$$




$$
\begin{aligned}
& y_{5,1}^{*}(\nu)=192 \tau^{15}-1188 \tau^{14}+3290 \tau^{13}-5450 \tau^{12}+6105 \tau^{11}-4947 \tau^{10}+ \\
& 2999 \tau^{9}-1365 \tau^{8}+455 \tau^{7}-105 \tau^{6}+15 \tau^{5}-\tau^{4}, y_{5,2}^{*}(\nu)=-360 \tau^{14}+ \\
& 2452 \tau^{13}-7576 \tau^{12}+14124 \tau^{11}-17796 \tau^{10}+16020 \tau^{9}-10556 \tau^{8}+5096 \tau^{7}- \\
& 1764 \tau^{6}+416 \tau^{5}-60 \tau^{4}+4 \tau^{3}, y_{5,3}^{*}(\nu)=-160 \tau^{13}+364 \tau^{12}+1246 \tau^{11}- \\
& 6730 \tau^{10}+13614 \tau^{9}-16170 \tau^{8}+12652 \tau^{7}-6796 \tau^{6}+2510 \tau^{5}-614 \tau^{4}+90 \tau^{3}- \\
& 6 \tau^{2}, y_{5,4}^{*}(\nu)=488 \tau^{12}-2668 \tau^{11}+5940 \tau^{10}-6420 \tau^{9}+2124 \tau^{8}+ \\
& 3152 \tau^{7}-4976 \tau^{6}+3520 \tau^{5}-1500 \tau^{4}+396 \tau^{3}-60 \tau^{2}+4 \tau, y_{5,5}^{*}(\nu)= \\
& 128 \tau^{11}-428 \tau^{10}+150 \tau^{9}+1250 \tau^{8}-2391 \tau^{7}+1837 \tau^{6}-461 \tau^{5}- \\
& 265 \tau^{4}+255 \tau^{3}-89 \tau^{2}+15 \tau-1, y_{5,6}^{*}(\nu)=-344 \tau^{10}+1820 \tau^{9}- \\
& 4000 \tau^{8}+4660 \tau^{7}-2976 \tau^{6}+864 \tau^{5}+80 \tau^{4}-140 \tau^{3}+40 \tau^{2}-4 \tau, \\
& y_{5,7}^{*}(\nu)=-32 \tau^{9}+100 \tau^{8}-14 \tau^{7}-334 \tau^{6}+600 \tau^{5}-488 \tau^{4}+210 \tau^{3}- \\
& 46 \tau^{2}+4 \tau, y_{5,8}^{*}(\nu)=-p_{3}^{*}(\nu), y_{6,1}^{*}(\nu)=-88 \tau^{16}+644 \tau^{15}-2152 \tau^{14}+ \\
& 4390 \tau^{13}-6170 \tau^{12}+6373 \tau^{11}-4999 \tau^{10}+3003 \tau^{9}-1365 \tau^{8}+455 \tau^{7}- \\
& 105 \tau^{6}+15 \tau^{5}-\tau^{4}, y_{6,2}^{*}(\nu)=192 \tau^{15}-1548 \tau^{14}+5742 \tau^{13}-13026 \tau^{12}+ \\
& 20229 \tau^{11}-22743 \tau^{10}+19019 \tau^{9}-11921 \tau^{8}+5551 \tau^{7}-1869 \tau^{6}+ \\
& 431 \tau^{5}-61 \tau^{4}+4 \tau^{3}, y_{6,3}^{*}(\nu)=-8 \tau^{14}+484 \tau^{13}-3356 \tau^{12}+10970 \tau^{11}- \\
& 21646 \tau^{10}+28562 \tau^{9}-26518 \tau^{8}+17732 \tau^{7}-8560 \tau^{6}+2926 \tau^{5}- \\
& 674 \tau^{4}+94 \tau^{3}-6 \tau^{2}, y_{6,4}^{*}(\nu)=-160 \tau^{13}+852 \tau^{12}-1422 \tau^{11}- \\
& 790 \tau^{10}+7194 \tau^{9}-14046 \tau^{8}+15804 \tau^{7}-11772 \tau^{6}+6030 \tau^{5}- \\
& 2114 \tau^{4}+486 \tau^{3}-66 \tau^{2}+4 \tau, y_{6,5}^{*}(\nu)=-40 \tau^{12}+172 \tau^{11}-272 \tau^{10}+ \\
& 330 \tau^{9}-946 \tau^{8}+2369 \tau^{7}-3451 \tau^{6}+3083 \tau^{5}-1765 \tau^{4}+651 \tau^{3}- \\
& 149 \tau^{2}+19 \tau-1, y_{6,6}^{*}(\nu)=128 \tau^{11}-772 \tau^{10}+1970 \tau^{9}-2750 \tau^{8}+ \\
& 2269 \tau^{7}-1139 \tau^{6}+403 \tau^{5}-185 \tau^{4}+115 \tau^{3}-49 \tau^{2}+11 \tau-1, \\
& y_{6,7}^{*}(\nu)=8 \tau^{10}-20 \tau^{9}-44 \tau^{8}+246 \tau^{7}-430 \tau^{6}+392 \tau^{5}-200 \tau^{4}+ \\
& 54 \tau^{3}-6 \tau^{2}, y_{6,8}^{*}(\nu)=-p_{2}^{*}(\nu), y_{7,1}^{*}(\nu)=32 \tau^{17}-276 \tau^{16}+1110 \tau^{15}- \\
& 2782 \tau^{14}+4890 \tau^{13}-6402 \tau^{12}+6431 \tau^{11}-5005 \tau^{10}+3003 \tau^{9}-
\end{aligned}
$$




$$
\begin{aligned}
& 1365 \tau^{8}+455 \tau^{7}-105 \tau^{6}+15 \tau^{5}-\tau^{4}, y_{7,2}^{*}(\nu)=-88 \tau^{16}+836 \tau^{15}- \\
& 3700 \tau^{14}+10132 \tau^{13}-19196 \tau^{12}+26602 \tau^{11}-27742 \tau^{10}+ \\
& 22022 \tau^{9}-13286 \tau^{8}+6006 \tau^{7}-1974 \tau^{6}+446 \tau^{5}-62 \tau^{4}+4 \tau^{3}, \\
& y_{7,3}^{*}(\nu)=64 \tau^{15}-804 \tau^{14}+4362 \tau^{13}-13862 \tau^{12}+29199 \tau^{11}- \\
& 43461 \tau^{10}+47349 \tau^{9}-38415 \tau^{8}+23283 \tau^{7}-10429 \tau^{6}+ \\
& 3357 \tau^{5}-735 \tau^{4}+98 \tau^{3}-6 \tau^{2}, y_{7,4}^{*}(\nu)=-8 \tau^{14}+324 \tau^{13}- \\
& 2504 \tau^{12}+9548 \tau^{11}-22436 \tau^{10}+35756 \tau^{9}-40564 \tau^{8}+ \\
& 33536 \tau^{7}-20332 \tau^{6}+8956 \tau^{5}-2788 \tau^{4}+580 \tau^{3}- \\
& 72 \tau^{2}+4 \tau, y_{7,5}^{*}(\nu)=32 \tau^{13}-316 \tau^{12}+1546 \tau^{11}-4842 \tau^{10}+ \\
& 10524 \tau^{9}-16384 \tau^{8}+18521 \tau^{7}-15259 \tau^{6}+9113 \tau^{5}-3879 \tau^{4}+ \\
& 1137 \tau^{3}-215 \tau^{2}+23 \tau-1, y_{7,6}^{*}(\nu)=-40 \tau^{12}+300 \tau^{11}- \\
& 1044 \tau^{10}+2300 \tau^{9}-3696 \tau^{8}+4638 \tau^{7}-4590 \tau^{6}+3486 \tau^{5}- \\
& 1950 \tau^{4}+766 \tau^{3}-198 \tau^{2}+30 \tau-2, y_{7,7}^{*}(\nu)=-12 \tau^{10}+ \\
& 86 \tau^{9}-274 \tau^{8}+515 \tau^{7}-641 \tau^{6}+563 \tau^{5}-361 \tau^{4}+169 \tau^{3}- \\
& 55 \tau^{2}+11 \tau-1, y_{7,8}^{*}(\nu)=-p_{1}^{*}(\nu), y_{8,1}^{*}(\nu)=-8 \tau^{18}+ \\
& 84 \tau^{17}-420 \tau^{16}+1330 \tau^{15}-2982 \tau^{14}+4998 \tau^{13}-6434 \tau^{12}+ \\
& 6435 \tau^{11}-5005 \tau^{10}+3003 \tau^{9}-1365 \tau^{8}+455 \tau^{7}-105 \tau^{6}+ \\
& 15 \tau^{5}-\tau^{4}, y_{8,2}^{*}(\nu)=32 \tau^{17}-364 \tau^{16}+1946 \tau^{15}-6482 \tau^{14}+ \\
& 15022 \tau^{13}-25598 \tau^{12}+33033 \tau^{11}-32747 \tau^{10}+25025 \tau^{9}- \\
& 14651 \tau^{8}+6461 \tau^{7}-2079 \tau^{6}+461 \tau^{5}-63 \tau^{4}+4 \tau^{3}, y_{8,3}^{*}(\nu)= \\
& -56 \tau^{16}+692 \tau^{15}-3928 \tau^{14}+13614 \tau^{13}-32258 \tau^{12}+ \\
& 55369 \tau^{11}-71075 \tau^{10}+69355 \tau^{9}-51701 \tau^{8}+29289 \tau^{7}- \\
& 12403 \tau^{6}+3803 \tau^{5}-797 \tau^{4}+102 \tau^{3}-6 \tau^{2}, y_{8,4}^{*}(\nu)= \\
& 64 \tau^{15}-812 \tau^{14}+4686 \tau^{13}-16366 \tau^{12}+38747 \tau^{11}-65897 \tau^{10}+ \\
& 83105 \tau^{9}-78979 \tau^{8}+56819 \tau^{7}-30761 \tau^{6}+12313 \tau^{5}-3523 \tau^{4}+ \\
& 678 \tau^{3}-78 \tau^{2}+4 \tau, y_{8,5}^{*}(\nu)=-56 \tau^{14}+668 \tau^{13}-3684 \tau^{12}+
\end{aligned}
$$




$$
\begin{gathered}
12414 \tau^{11}-28478 \tau^{10}+46928 \tau^{9}-57140 \tau^{8}+52081 \tau^{7}- \\
35591 \tau^{6}+18069 \tau^{5}-6667 \tau^{4}+1717 \tau^{3}-287 \tau^{2}+27 \tau-1 \\
y_{8,6}^{*}(\nu)=32 \tau^{13}-356 \tau^{12}+1846 \tau^{11}-5886 \tau^{10}+12824 \tau^{9}-20080 \tau^{8}+ \\
23159 \tau^{7}-19849 \tau^{6}+12599 \tau^{5}-5829 \tau^{4}+1903 \tau^{3}-413 \tau^{2}+ \\
53 \tau-3, y_{8,7}^{*}(\nu)=-8 \tau^{12}+92 \tau^{11}-480 \tau^{10}+1506 \tau^{9}-3170 \tau^{8}+ \\
4721 \tau^{7}-5103 \tau^{6}+4033 \tau^{5}-2311 \tau^{4}+935 \tau^{3}-253 \tau^{2}+41 \tau-3 \text { and } \\
y_{8,8}^{*}(\nu)=-p_{0}^{*}(\nu)+(\tau-1)^{11}
\end{gathered}
$$

Among the results of calculations (90)-(91)-(92) we emphasize the following

$$
y_{i, 8}^{*}(\nu)=-p_{8-i}^{*}(\nu) \text { for } i \in[1,7] \cap \mathbb{Z} .
$$

\section{3 End of the Proof of Theorem ??1}

Let $\tilde{B}(z ; \nu)$ be $8 \times 8$-matrix such that $-(z-1)^{-1} z \bar{R}(\nu)$ is its last row, and all elements of its other rows are equal to 0 . Let $B(z ; \nu)=\left(N_{8}+\tilde{B}(z ; \nu)\right)$. In view of (26), (37),

$$
\delta X_{k}(z, \nu)=B(z ; \nu) X_{k}(z ; \nu)
$$

Let $\bar{s}_{i}^{*}(\nu)=\sum_{k=1}^{8} s_{i, k}^{*}(\nu), \bar{v}_{i}^{*}(\nu)$ and $\bar{a}_{i}^{*}(z, \nu)=\sum_{k=1}^{8} a_{i, k}^{*}(z, \nu)$ be the $i$-th row of the matrices respectively $S^{*}(\nu), V^{*}(\nu)$ and $A^{*}(z, \nu)$. In view of (30), (32),

$$
\begin{gathered}
\bar{s}_{i}^{*}(\nu) N_{8}=s_{i+1}^{*}(\nu) \text { for } i \in[1,7] \cap \mathbb{Z}, \\
a_{i, k}^{*}(z, \nu)=s_{i, k}^{*}(\nu)+z v_{i, k}^{*}(\nu), \bar{a}_{i}^{*}(z, \nu)=\bar{s}_{i}^{*}(\nu)+z \bar{v}_{i}^{*}(\nu)
\end{gathered}
$$

with $\{i, k\} \subset[1,8] \cap \mathbb{Z}$, and in view, of $(93)$, if $i \in[1,7] \cap \mathbb{Z}$, then

$$
a_{i, 8}^{*}(z, \nu)=s_{i, n}^{*}(z \nu)+z v_{i, n}^{*}(z, \nu)=p_{8-i}(1-z) .
$$

We must prove the equality

$$
\nu^{11} \delta^{i-1} f_{k}(z, \nu-1)=\bar{a}_{i}^{*}(z, \nu) X_{k}(z, \nu) \text { if } i \in[1,8] \cap \mathbb{Z}, k \in \mathfrak{K}, \nu \in \mathfrak{M}_{1}
$$


Since $T^{*}(z, w, \nu)=\sum_{k=0}^{7}\left(p_{k}^{*}(\nu)+z q_{k}^{*}(\nu)\right) w^{k}$, the equality (97) for $i=1$ follows from (26) and (47). Suppose that (97). holds for some $i \in[1,7] \cap \mathbb{Z}$. Then in view of (97), (32), (94), (96), (88), (95),

$$
\begin{gathered}
\nu^{11} \delta^{i} f_{k}(z, \nu-1)=\delta\left(\bar{a}_{i}^{*}(z, \nu) X_{k}(z, \nu)\right)=\left(\delta \bar{a}_{i}^{*}(z, \nu)\right) X_{k}(z, \nu)+ \\
\bar{a}_{i}^{*}(z, \nu) \delta X_{k}(z, \nu)=\left(z \bar{y}_{i}^{*}(\nu)+\bar{a}_{i}^{*}(z, \nu)\left(N_{8}+\tilde{B}(z ; \nu)\right) X_{k}(z, \nu)=\right. \\
\bar{s}_{i}^{*}(\nu) N_{8} X_{k}(z, \nu)+\left(z \bar{y}_{i}^{*}(\nu)\left(E_{8}+N_{8}\right)+p_{8-i}(1-z) \tilde{B}(z ; \nu)\right) X_{k}(z, \nu)= \\
\left(z \bar{y}_{i}^{*}(\nu)\left(E_{8}+N_{8}\right)+z \bar{a}_{i}^{*}(z, \nu) \tilde{B}(z ; \nu) p_{8-i}(1-z)\left(-(z-1)^{-1} z \bar{R}(\nu)\right)\right) X_{k}(z, \nu)+ \\
\bar{s}_{i+1}^{*}(\nu) X_{k}(z, \nu)=\left(z \bar{y}_{i+1}^{*}(\nu)+\bar{s}_{i+1}^{*}(\nu)\right) X_{k}(z, \nu) . \square
\end{gathered}
$$

\section{References}

[1] R.Apéry, Interpolation des fractions continues et irrationalite de certaines constantes, Bulletin de la Section des Sciences du C.T.H., No. 3 (1981), 37-53.

[2] Bateman Harry, Erdelyi Arthur, Higher Transcendental Functions, Volume 1, McGrawHill Book Company, Inc., 1953.

[3] L.A. Gutnik, On some systems of difference equations, Chebyshevskij Sbornik (Chebyshev Collection), 7, No. 3 (2006).

[4] L.A. Gutnik, On a difference system for some Mejer's funtions, International Journal of Pure and Applied Mathematics, 89, No. 3 (2013), 409-432, doi: 10.12732/ijpam.v89i3.12.

[5] L.A. Gutnik, On expansion of Zeta(3) in continued fraction, International Mathematical Forum, 8, No. 16 (2013), 771-781, doi: 10.12988/imf.2013.13081.

[6] L.A. Gutnik, On expansion of Zeta(3) in continued fraction, Part 2, International Mathematical Forum, 8, No. 28 (2013), 1385-1396, doi: 10.12988/imf.2013.137137.

[7] L.A. Gutnik, On Some Systems of Difference Equations, MPI für Mathematik, Bonn, Preprint Series, Part 1: 2006, 23, 1 - 37; Part 2: 2006, 49, 1 - 31, Part 3: 2006, 91, 1 52; Part 4: 2006, 101, 1-49, Part 5: 2006, 115, 1 - 9; Part 6: 2007, 16, 1 -30, Part 7: 2007, 53, 1-34; Part 8: 2007, 64, 1 - 44, Part 9: 2007, 129, 1 -36; Part 10: 2007, 131 , $1-33$, Part 11: 2008, $38,1-45$; Part 12: 2008, 55, $1-45$. 